\title{
Hollow structures as drug carriers: Recognition, response, and release
}

\author{
Decai Zhao ${ }^{1,2}$, Nailiang Yang ${ }^{1,2}(\bowtie)$, Lekai $X u^{1,3}$, Jiang $\operatorname{Du}^{3}$, Yang Yang ${ }^{4,5}(\bowtie)$, and Dan Wang ${ }^{1,2}(\bowtie)$ \\ ${ }^{1}$ State Key Laboratory of Biochemical Engineering, Institute of Process Engineering, Chinese Academy of Sciences, Beijing 100190, China \\ ${ }^{2}$ University of Chinese Academy of Sciences, Beijing 100049, China \\ ${ }^{3}$ Green Catalysis Center, and College of Chemistry, School of Materials Science and Engineering, Zhengzhou University, Zhengzhou 450001 China \\ ${ }^{4}$ Department of Thoracic Surgery, Shanghai Pulmonary Hospital, School of Medicine, Tongji University, Shanghai 200433, China \\ ${ }^{5}$ School of Materials Science and Engineering, Tongji University, Shanghai 201804, China
}

(C) Tsinghua University Press and Springer-Verlag GmbH Germany, part of Springer Nature 2021

Received: 2 April 2021 / Revised: 14 May 2021 / Accepted: 15 May 2021

\begin{abstract}
Hollow structures have demonstrated great potential in drug delivery owing to their privileged structure, such as high surfaceto-volume ratio, low density, large cavities, and hierarchical pores. In this review, we provide a comprehensive overview of hollow structured materials applied in targeting recognition, smart response, and drug release, and we have addressed the possible chemical factors and reactions in these three processes. The advantages of hollow nanostructures are summarized as follows: hollow cavity contributes to large loading capacity; a tailored structure helps controllable drug release; variable compounds adapt to flexible application; surface modification facilitates smart responsive release. Especially, because the multiple physical barriers and chemical interactions can be induced by multishells, hollow multishelled structure is considered as a promising material with unique loading and releasing properties. Finally, we conclude this review with some perspectives on the future research and development of the hollow structures as drug carriers.
\end{abstract}

\section{KEYWORDS}

hollow structure, drug delivery system, carriers, spatiotemporal

\section{Introduction}

Over the past few decades, the increasing global concerns regarding disease treatment and medication safety have aroused awareness of the drug delivery system (DDS) [1-4]. Considering the unique characteristics of different materials, and their specific advantages in terms of biocompatibility, stability, and intelligent controlled release, a great number of candidates such as liposomes, polymers, silicon-based materials, hydroxyapatite, metal oxides, and biological macromolecules have been explored [5-8]. A rational structural design on drug carriers can modify the physical and chemical interaction between the drug molecules and the carrier, which is critically important for a drug delivery system.

In the 1960s, Folkman et al. developed silicone rubber as a drug carrier, which prolonged the effective therapy of the drug and aroused much interest on the drug delivery system [9]. In 1974, Hiroshi et al. fabricated hollow $\mathrm{ZnO}$ [10], which initiated the era of hollow materials. Following this, research on hollow structures with micro/nano scale as drug carriers sprung up. Years of studies have proved that hollow nanostructures materials are advantageous for drug delivery due to qualities such as high surface-to-volume ratio, low density, and large cavities. The hollow structure serves as a drug reservoir and can promote the sustained drug release, keeping the drug effective for a long period. Hollow cavities are beneficial for high payloads and the shell can provide a physical barrier.
The primary mission of an ideal drug delivery system is to release enough of the drugs it carries. Besides, recognizing the targeted system, and responding to environmental stimuli would make the DDS smarter. In this case, drugs can be released at the targeted site and reach an effective concentration, as well as reduce leakage at non-target sites.

Among the various factors in the drug delivery system, to understanding the choice of carrier, drug loading, and release method, the driving forces and the interactions need to be deeply understood. Therefore, the design at molecular level is critical to DDS. From single shelled hollow structure to hollow multishelled structure, the complex architecture would provide abundant space for drug loading and more barriers for drug delivery, which would result in a smarter system. Consequently, we need to systematically understand the series of processes and interactions of the drug delivery system.

In this review, we firstly introduce the structure of the carrier materials which have been applied to DDS, including hollow mesoporous structures, hollow single-shell structures, hollow multi-shell structures, and Janus particles. Later, we introduce various drug loading approaches and the interactions between hosts and guests. Subsequently, we respectively introduce the processes of drug carrier action, namely recognition, response, and release (Fig. 1) with hollow structures. Beginning with an introduction of the recognition, we give the strategies for passive targeting and active targeting, respectively. Then, the various responses to endogenous and exogenous stimulus 


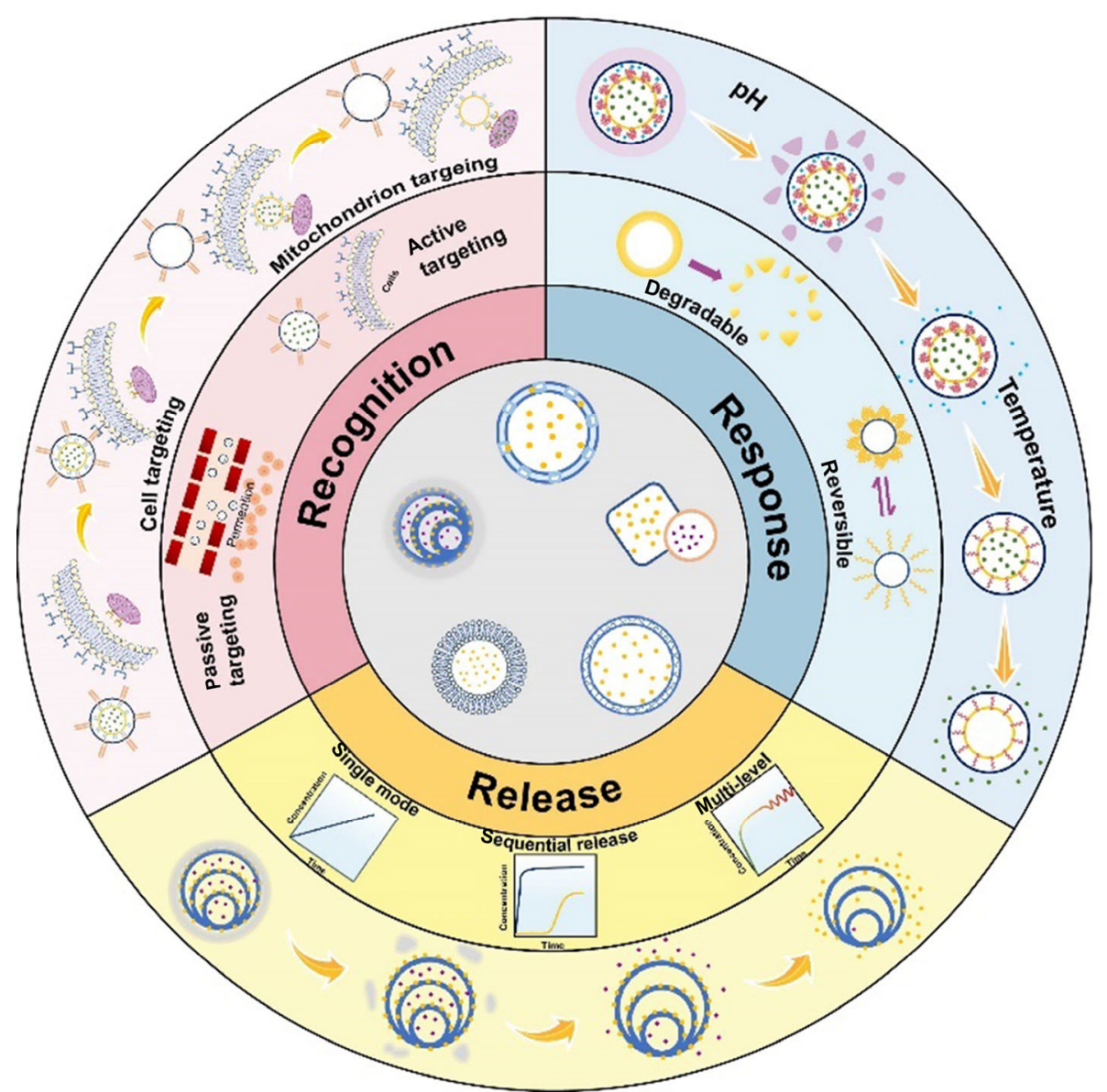

Figure 1 Illustration of hollow structures applied in drug delivery system. Hollow carrier: Various structures with hollow structure. The middle circle includes recognition: active targeting and passive targeting; response: degradable carrier and structurally reversible carrier under stimulus; release: schematic curve of single mode, multi-level, and sequential release. The outer circle presents the unique sequential targeting, sequential response, and multi-level drug release by hollow multishelled structures.

are summarized. In the part of release, some release modes and corresponding strategies for constructing are presented. At the end of this review, some challenges of the hollow nanostructures in drug delivery system and the outlook in material design with hollow multishelled structures are proposed.

\section{The structures of hollow carriers}

Carrier is primely important for the drug delivery system, and its structure has great influences on the release performance of their loads. The structure affects the path and driving force of the drug molecule in diffusing outward, due to the pore size, the number and thickness of the shells, and the structural symmetry. Based on these structures, various components (e.g. organic, inorganic, or composition) affect the interaction between drugs and carriers, and the behavior changes in the release environment (Fig. 2).

\subsection{Hollow mesoporous structure}

Mesoporous materials refer to a type of porous material with pores between 2 and $50 \mathrm{~nm}$, which possess the unique characteristics, such as ultra-high specific surface area, large pore volume, adjustable pore size and morphology [11]. They have potential advantages in the fields, including drug delivery, electrocatalysis [12], and photocatalysis [13].

With the prospering of research on hollow structures, researchers have hollowed out porous materials to further improve their performance as drug carriers. The preparation (a)

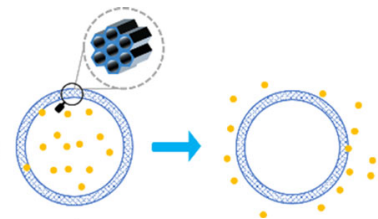

(b)

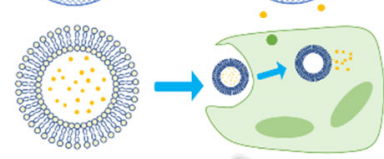

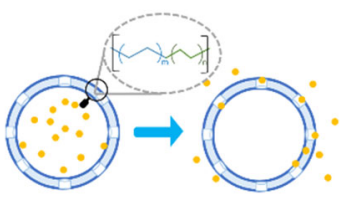

(c)

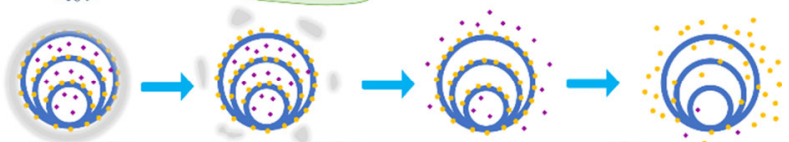

(d)
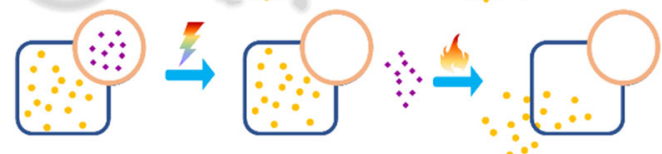

Figure 2 Illustration of different carriers and release characteristics of drug molecules in (a) hollow mesoporous material, (b) hollow liposome and polymer capsule, (c) hollow multi-shelled structure, and (d) Janus particle.

of hollow materials usually uses oil droplets, crystals, hard microspheres as templates [14-16], after the precursor is selfassembled or coated on the microspheres, and the template is removed by high-temperature pyrolysis, etching, etc., to obtain hollow silicon materials [17].

Among them, mesoporous silica is widely utilized in the drug delivery system, owing to its stable skeleton structure, easily modified surface, and good biocompatibility [7, 18-21]. 
In 2005, Shi et al. increased the ibuprofen loading capacity to $918.6 \mathrm{mg} \cdot \mathrm{g}^{-1}$ through the design of the hollow structure of mesoporous silica [22], which is three times higher than that of the conventional mesoporous materials [23].

Drug release from hollow mesoporous silica (HMS) was controlled by diffusion and sometimes the release profile can be fitted by the Higuchi model $[24,25]$. Some researchers have adjusted the structure to control the release rate of drugs, such as pore-size and shell thickness [26]. As shown in Fig. 3(a), Li et al. [25] found that the loading capacity of hollow mesoporous silica with a uniform hollow core increased along with the decreasing thickness of the shell, and meanwhile, the release rate increased as the shell thickness became thinner.

In addition to thickness of shells, the pore size also influence release property. $\mathrm{Li}$ and co-workers [27] constructed a series of hollow mesoporous silica nanoparticles (HMSNs) with pore sizes of 3.2, 6.4, and $12.6 \mathrm{~nm}$. The loading efficiency increased with the pore size, and the release rates exhibited pore-sizedependent characteristics both in vitro and intracellular drug release experiments (Fig. 3(b)). Similar conclusions have appeared in other reports $[25,28]$.

The modification of carrier materials by surface engineering is of great significance for regulating the surface electrical properties, hydrophilicity, and dispersibility of the carrier, thereby improving the drug delivery performance [29]. Typically for the porous silicon materials, apart from traditional oxidation, thermal carbonization, hydrosilylation, and other ways to stabilize and functionalize the surface, it also includes strategies such as coating polymer gating on the outer layer, surface grafting molecules to control the phase transition temperature, and pore modification [30].

Although there are various methods of surface engineering, one should consider the interaction force between the drug molecule and the carrier, and the interaction between carriers and cells or microorganisms, so as to strengthen the ability of controlled release, physiological stability, cell targeting properties [31]. For example, researchers can adjust the surface roughness of a material to control its biological properties such as cell capture and adhesion [32,33]. Song et al. constructed a hollow pollen-like mesoporous silica material. The rough surface topology increases the adhesion to bacteria, thus exhibiting enhanced lysozyme transfer characteristics and antibacterial activity [34].

Modifying the inorganic materials with polymers is another common strategy of surface engineering. Zhu et al. covered polyelectrolyte multilayer coatings on HMS. Results showed that the carrier had a smaller loading capacity and a slower release rate, which can be contributed to the double barrier provided by the polymer multilayers and the mesoporous layer [35].

In addition to coating the mesoporous silica with polymers $[36,37]$, other functional materials such as nanoparticles $[38,39]$, and metal-organic frameworks (MOFs) [40], can also improve the controlled release of drugs and be introduced as smart response by compounding with silica. Jia et al. fabricated a DOX/HMS@ZIF capsule (DOX = doxorubicin) as drug carrier by coating ZIF on the outer surface of HMS [40]. After that, the loading capacity of DOX can reach up to $44 \mathrm{wt} . \%$. (a)
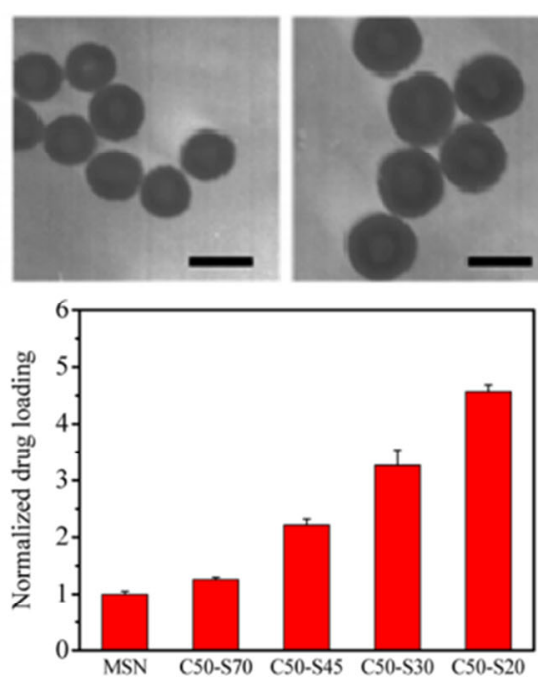

(b)

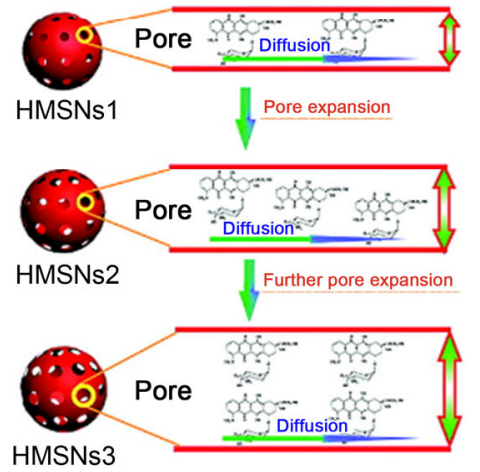

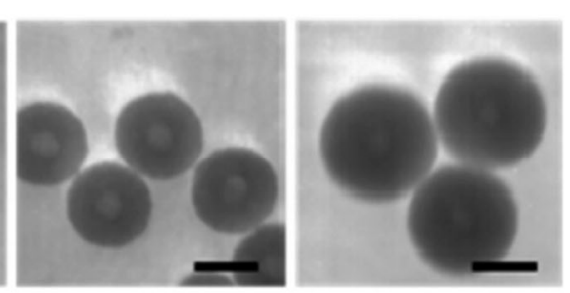
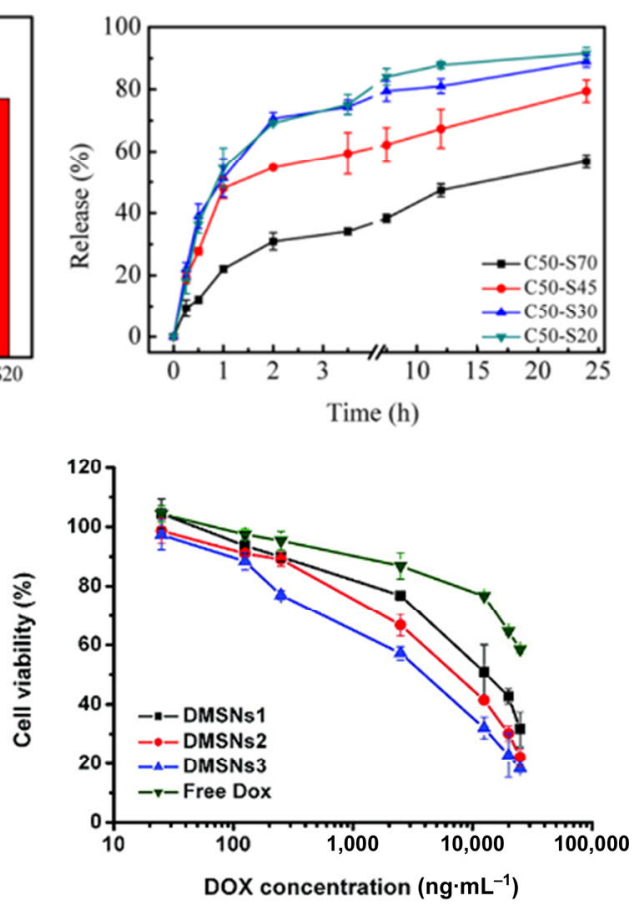

Figure 3 (a) Schematic illustration for the preparation of HMSNs (scale bars are $100 \mathrm{~nm}$ ) and their use in drug loading and release (reproduced with permission from Ref. [25], () American Society of Chemical 2016). (b) Representative schematic illustration of controlling DOX release rate by tuning the pore sizes of HMSNs to 3.2, 6.4, and $12.6 \mathrm{~nm}$, and in vitro cytotoxicity of DMSNs with different pore sizes against MCF-7 and MCF-7/ADR cells (reproduced with permission from Ref. [27], () American Society of Chemical 2011). 
Hollow mesoporous organosilica nanoparticles (HMONs) not only inherit the advantages of mesoporous silica, but also construct an organic-inorganic hybrid structure, and they have attracted widespread attention in the field of biomedicine [41-44]. Compared with inorganic mesoporous silica, the organic groups in HMONs are homogeneously and covalently incorporated in the pore at the molecular level, which endow them with versatile organic-inorganic hybrid frameworks, and therefore bring improved biocompatibility, enhanced thermal stability and controllable wetting ability [45]. Chen et al. developed a new generation HMONs with multiple organic groups as DOX carriers. The incorporation of thioether can response to the reduction microenvironment by disulfide bonds, and its easily-cleaved property by ultrasound endows HMONs with high intensity focused ultrasound (HIFU)-responsive drug release performances. In addition, the organosilica carrier has a higher biocompatibility, tumor accumulations and enhanced therapeutic efficiency against cancer [46].

Moreover, the hetero hydrophobic/hydrophilic composition in HMONs can be designed to adapt the environment in vivo. Yang et al. noted that compared with inorganic silica, the HMONs showed the better sustained release property owing to the strong hydrophobic interaction between HMONs and the guest when using them as antigen carriers [47]. In addition, the intrinsic hydrophobicity of HMONs endues them with stronger interaction with antigen presenting cells, resulting in endolysosomal escape.

Apart from silicon-based materials, carbon nanomaterials show great application prospects in drug delivery, owing to their high specific surface area, long residence time in vivo, good biological inertness, high cell uptake efficiency, and excellent drug release characteristics [48]. Ahmad et al. reported mono-dispersed mesoporous hollow carbon nanospheres for the delivery of antimicrobials vancomycin [49]. By designing mesoporous hollow carbon (MHC) materials with different pore sizes and wall thicknesses, the MHC materials possess a vancomycin loading capacity of $861 \mathrm{mg} \cdot \mathrm{g}^{-1}$. Initial fast release followed by sustained release of drugs displayed excellent antibacterial performance.

\subsection{Hollow single-shell structure}

\subsubsection{Liposome}

Liposomes are composed of phospholipid bilayers. The hydrophobic tails of phospholipid molecules tend to gather to avoid the water, while the hydrophilic heads are exposed to the water phase and form closed vesicles with a bilayer structure. Liposomes have been widely utilized in the drug delivery system since the 1960s owing to their excellent targeting, stability, biocompatibility and ease of modification $[50,51]$. With the development of hollow structure, liposomes have drawn more attention from researchers. In clinical trials, liposome drug delivery systems have been applied to the targeted delivery of various anti-cancer drugs including both hydrophobic and hydrophilic drugs.

Transdermal diffusion and cell fusion are two methods of which liposome carriers can release drugs. With liposome gels for external use, drug molecules enter the skin through transdermal diffusion. Kirjavainen et al. studied the influence of phospholipid liposomes on the skin permeability of model drugs, and the results indicated that permeabilities of certain model drugs were substantially increased when ethanol was present in donors with eggyolk lecithin [52]. While oral liposomes are used, the drug usually adheres to and fuses with the cells in the gastrointestinal tract to release the drug [53].

There are liposomes that have special properties, such as temperature-sensitivity, $\mathrm{pH}$-sensitivity, and light-sensitivity, which endows them with corresponding responsive release of drugs. The report introduces a temperature-sensitive liposome drug delivery system [54]. The liposome is loaded with superparamagnetic iron oxide nanoparticles. Because the composite can increase the localized temperature by the alternating magnetic field, the structure transition takes place on the thermosensitive liposome to help realizing the controlled release of DOX. Accompany with the advantages of transport across a blood-brain barrier and selectively target for brain glioma, the composite displays a higher cellular uptake property (Fig. 4(a)).

\subsubsection{Polymeric capsules}

Another subset of hollow organic materials, polymeric capsules, have been developed as carriers for a variety of drugs, enzymes, genes, and proteins [55]. Their polymeric shell can increase the stability of loaded proteins and nucleic acids, and protect them from degradation by proteases and nucleases. Besides, the properties of nanocapsules can be adjusted by screening different functional monomers or cross-linking agents, such as surface charge, long cycling and degradability. There are common polymers, such as polylactic acid (PLA), poly(lacticco-glycolic acid) (PLGA) [56-58], that are widely used in drug
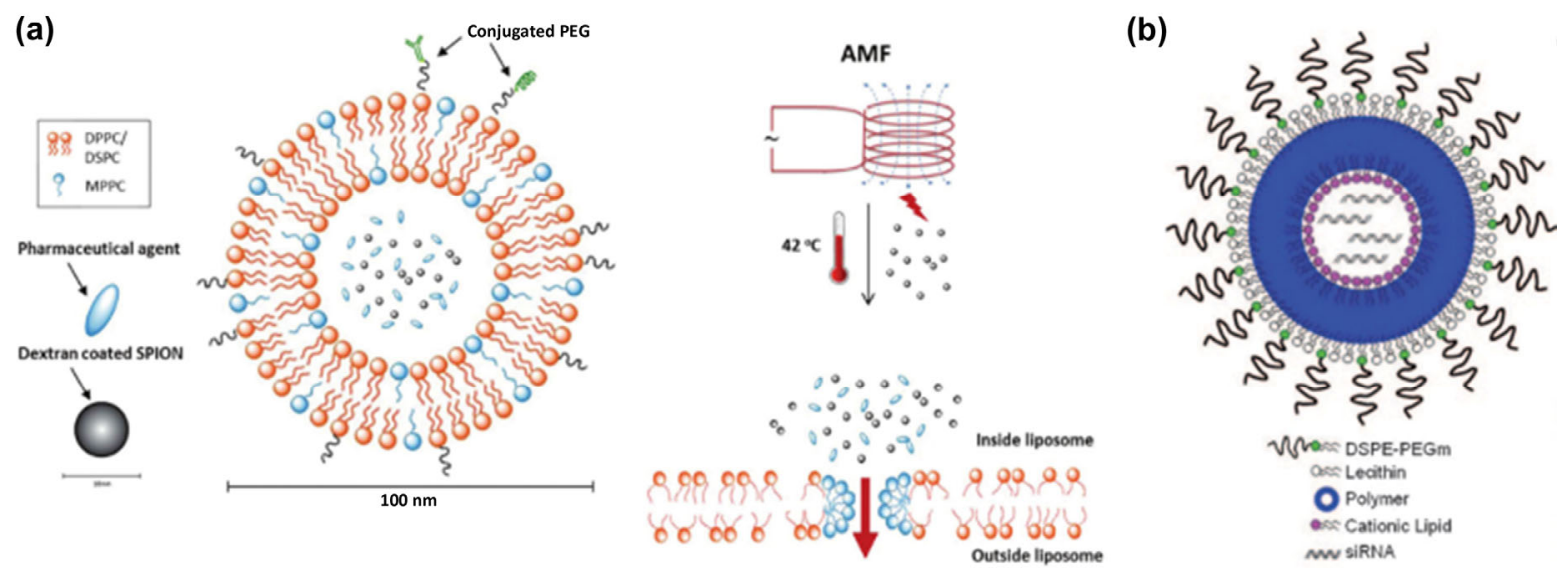

Figure 4 (a) Illustration of alternating magnetic field (AMF) induced drug release from DOX@P1NS/TNC-FeLPs (reproduced with permission from Ref. [54], ( ) The Royal Society of Chemistry 2019). (b) Schematic representation of the lipid-polymer-lipid hybrid nanostructure. The particle is composed of an outer lipid-PEG surface, a middle polymer layer, and an inner cationic lipid hollow core entrapping aqueous siRNA (reproduced with permission from Ref. [63], (c) WILEY-VCH Verlag 2011). 
delivery systems and have good biocompatibility and stability [59]. For non-degradable polymer materials, drug release is determined by a concentration gradient. In contrast, the release for degradable polymer carriers is controlled by both the molecular diffusion rate and the carrier degradation rate [60]. It would also be beneficial to develop smart hollow polymer carriers with environmental response, such as temperature $[33,61]$ and $\mathrm{pH}$ response $[56,62]$. Lee and coworkers constructed a hollow porous PLGA microsphere as a drug carrier for dexamethasone (DEX) [56]. Owing to the high permeability, the DEX encapsulation and loading efficiencies were $70 \%$ and $0.7 \%$. Here, the release is controlled via two mechanisms: concentration diffusion and PLGA microsphere erosion and degradation.

Liposomes and polymers are distinctive, and their combination results in surprising discoveries. Farokhzad et al. developed a hollow lipid-polymer-lipid hybrid nanoparticle as RNA carrier [63]. The encapsulation efficiency was approximately $82 \%$. Compared to single-component polymer, the RNA encapsulation efficiency increased by more than 10 times (Fig. 4(b)).

\subsubsection{Metal-organic frameworks}

Metal-organic framework is a powerful participant in drug carriers. Researchers compiled some comprehensive reviews, which summarized the applications of MOFs in drug delivery and cancer therapy over the past decades $[4,64,65]$.

The higher porosity and larger specific surface areas of hollow MOFs than solid counterparts endow the opportunity for the efficient adsorption and storage of drug molecules [66-68]. Tian and co-workers constructed a multifunctional platform by using a hollow porphyrinic meal-organic framework (H-PMOF) nanoparticle as the drug carrier [67]. DOX and indocyanine green were loaded with an ultrahigh loading capacity of $635 \%$, higher than that of the nonhollow PMOF. Furthermore, the photodynamic therapy was more effective owing to a shorter diffusion distance of the generated $\mathrm{O}_{2}$.

\subsection{Hollow multishell structure (HoMS)}

HoMS is an assembly of multiple shells with voids between the individual shells. Compared with hollow spheres, HoMS has more interfaces, a larger specific surface area, and a multi-stage cavity structure, and is widely used in energy conversion and storage, catalysis, drug sustained release, and other fields [69-71]. HoMS has attracted more attentions in drug delivery systems for their special hierarchical structures, which significantly prolong the duration of drug release.

The driving force of drugs released from HoMS is mainly controlled by the concentration gradient. The unique multishell structures contribute to the sustained release by hindering the diffusion of drug molecules. Ma et al. showed that hollow multishelled porous $\mathrm{CaCO}_{3}$ possessed a high drug loading capacity with $245.33 \mu \mathrm{g} \cdot \mathrm{mg}^{-1}$ and a sustained-release drug profile up to $115 \mathrm{~h}$ [72]. The drug release kinetics were fitted to the Higuchi model and characterized with three stages due to the hierarchical shell and multi-level tunnel structures.

Silicon materials with a hollow multi-shell structure, which combine the advantages of the multi-level release from HoMS and the biocompatibility from silicon, can be utilized in cancer therapy $[73,74]$. Teng et al. prepared a multi-shelled periodic mesoporous organosilica (PMO) hollow sphere which was perfectly biocompatible for human breast cancer (MDA-MB-231) cells and killed $84 \%$ of cells within $48 \mathrm{~h}$ at a DOX concentration of $4 \mu \mathrm{g} \cdot \mathrm{mL}^{-1}$ (Fig. 5(a)) [75].

Notably, spatiotemporal ordering was found to be a unique property of HoMS in drug release. Since HoMS can provide different storage sites and various interactions for drug molecules, (a)

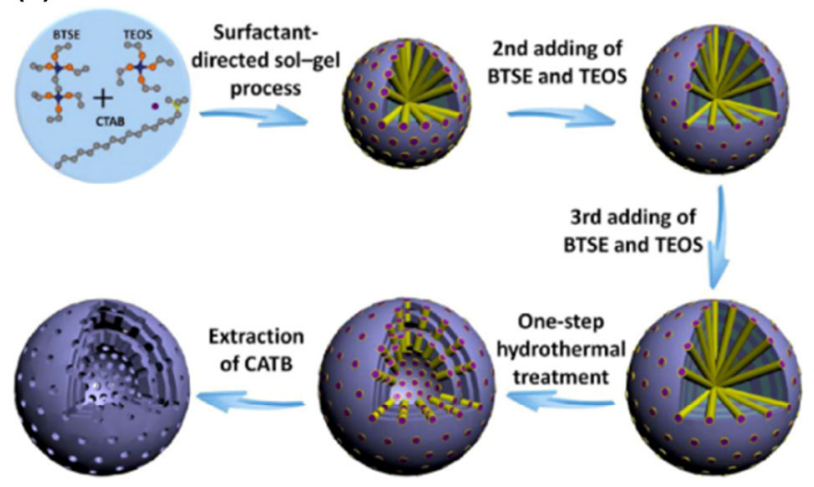

(b)

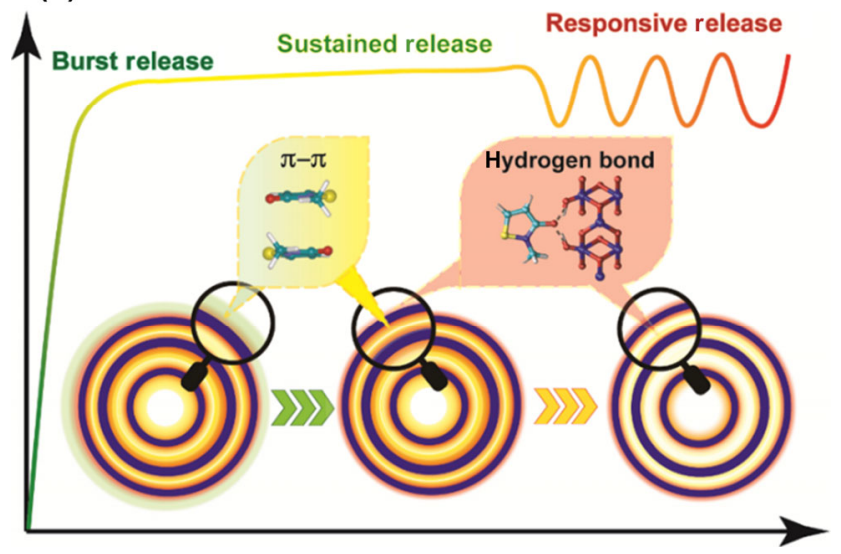

Figure 5 (a) Illustration of the preparation of triple-shelled PMO hollow spheres by the multi-interface transformation approach (reproduced with permission from Ref. [75], ( ) American Chemical Society 2015). (b) Scheme of the sequential drug release of hollow multi-shelled structures (reproduced with permission from Ref. [76], (c) Zhao, D. C. et al. 2020).

the release also shows the time sequence accordingly. As shown in Fig 5(b) [76], the drugs adsorbed on the surface are easily released at the initial stage, resulting in burst release; the drugs in the cavity through $\pi-\pi$ stacking interactions contribute to the following sustained release stage and also the responsive release stage; the drug molecules that form hydrogen bonds with the surface also contribute to part of the responsive release. Therefore, it is a multi-level release process induced by physical barriers and chemical interaction.

HoMS has many advantages in drug sustained release, but it is still in the early stages of development for application in this field. As an example, heterogeneous HoMSs with different inner and outer layer components can be loaded with different drugs by adjusting the surface properties to achieve sequential sustained release of multiple drugs.

\subsection{Janus particles}

Janus particles have attracted significant attention as smart drug carriers owing to their unique dual properties. Janus particles can be synthesized with diversified forms (e.g., cauliflower-like, dumbbell-like, and core-shell towards cauliflower-like structures) and used in the delivery of two cargoes simultaneously.

The release behavior is significantly affected by the structure of composite particles [77-79]. Janus particles are allowed to collect two completely different chemicals, such as hydrophobicity, polarity, functionality, and surface charge differences, as a result of their asymmetrical and unique structure. The existence of two distinct faces makes these particles attractive colloids that can be utilized to simultaneous detection and treatment of cells [80]. These biocompatible Janus nanoparticles have a bipolar affinity to human cells and can carry a drug and detect the targeted 
cells at the same time [81]. Simultaneous drug delivery and cell detection result in Janus particles being super-smart and multifunctional.

A dual-compartment Janus mesoporous silica nanocomposite (UCNP@SiO ${ }_{2} @ \mathrm{mSiO}_{2} \& \mathrm{PMO}$ ) was fabricated by Li et al. [78], and used as the carrier for both hydrophobicity/hydrophilicity dual guests, which can be further applied into nano biomedicine for heat and NIR light bimodal triggered dual drugs release (Fig. 6(a)).

In addition to the overall structural asymmetry, the morphology can also be constructed as a Janus particle which is symmetrical on the outside but has a difference in hydrophilicity and hydrophobicity on the inside. Chen et al. reported a Tai Chi-like ultrasmall nanoparticles-stacked oleic acid-NaYF4:Yb,Er@hollow porous $\mathrm{SiO}_{2}$ [82], which resembled a symmetrical silicon hollow sphere from the outside, and embodied a hydrophobic interior for water insoluble drugs and a hydrophilic $\mathrm{SiO}_{2}$ surface for water soluble guests, offering an efficient combination therapy (Fig. 6(b)).

\section{The interaction between drug and hollow}

\section{carriers}

Besides the component and structure of carrier materials, the drug-loading method and the carrier-drug interaction play important roles in the behavior of the delivery system.

\subsection{Drug loading}

An outstanding drug release system should have a large drug loading capacity to reduce the dosage of carriers. Different drug loading mechanisms affect the interaction between host and guest and determine it can be effectively released in the required environment.

For efficient drug action, improving the drug loading efficiency is critical in drug carrier research. The combination of drug carriers and drugs is related to their intrinsic properties as well as the method of drug loading.

The selection of drug loading method is intricately connected to the synthesis of carriers and the properties of drug molecules, and also has influences on the behaviors of drug release. In view of the special approach of the preparation of hollow structure materials, the strategies are usually divided into preloading and post loading.

\subsubsection{Pre-loading Strategies}

The pre-loading method means that the drug molecules are loaded prior to the formation of carriers. Generally, the precursors of carriers are directly immersed in the drug solution, and the concentration gradient drives the drug into the carrier. As an example, Zhang et al. mixed a certain amount of methylene blue (MB) and tetraethyl orthosilicate (the precursor of $\mathrm{SiO}_{2}$ ) and stirred the solution for $24 \mathrm{~h}$ to obtain MB-loaded $\mathrm{SiO}_{2}$ microspheres [83].

The solvent evaporation approach is to dissolve the drug and the polymer in a volatile organic solvent which is immiscible with water, ultrasonically emulsify the solution and finally the solvent is evaporated to form polymer nanoparticles. Sung et al. used the $\mathrm{W}_{1} / \mathrm{O} / \mathrm{W}_{2}$ preparation method to load the hydrophobic drug rifampicin into PLGA microspheres [84], which effectively solved the problem of low loading efficiency of hydrophobic drugs, increasing the entrapment efficiency to $73.4 \%$.

For hollow structures prepared by the template method, the pre-loading approach sometimes refers to loading the drug on a template and then coating other substances on the outside [85]. Zhao et al. presented a mild method of preloading the DOX in $\mathrm{CaCO}_{3}$ sphere and then coated with mesoporous silica [86]. After the template of $\mathrm{CaCO}_{3}$ was removed, the drug was stored in the cavity. This method could bring a higher encapsulation efficiency and provide a new idea for nano-sized shapecontrollable hollow drug therapeutics.

\subsubsection{Post-loading strategies}

The post loading method refers to loading the drug after the carriers have been synthesized.

The solvent adsorption approach means immersing the prepared carriers in the drug solution, and the drug is adsorbed on the surface or in the cavity of the materials. Since the drug concentration outside the carrier is higher than inside, the drug molecules diffuse into it under the driving force of the (a)
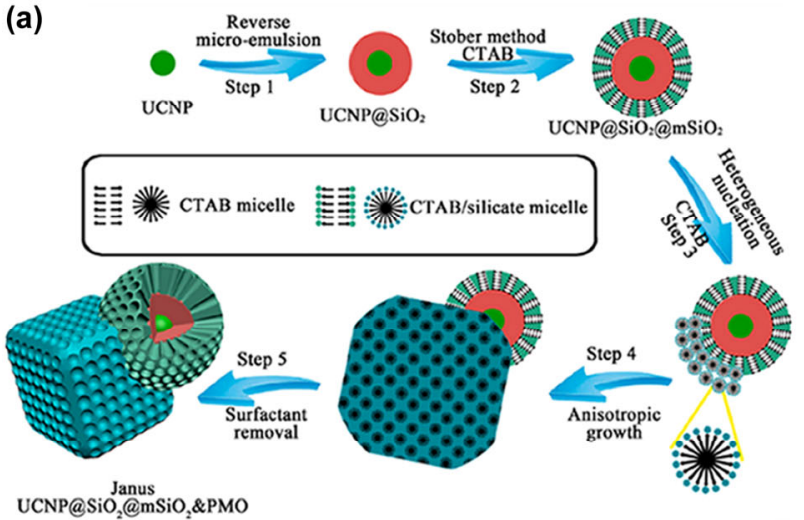

UCNP@SiO ${ }_{2} @ \mathrm{mSiO}_{2} \& \mathrm{PMO}$
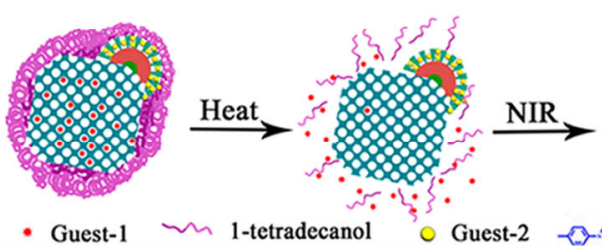

(b)
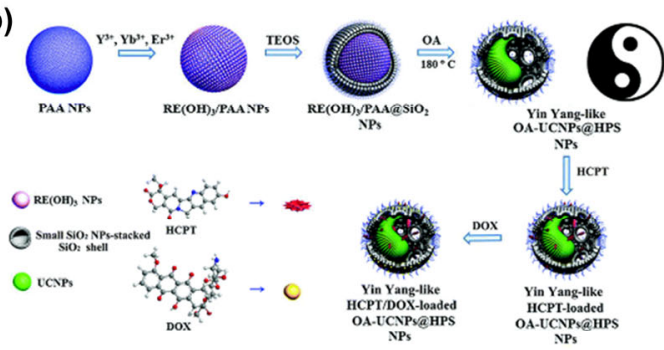

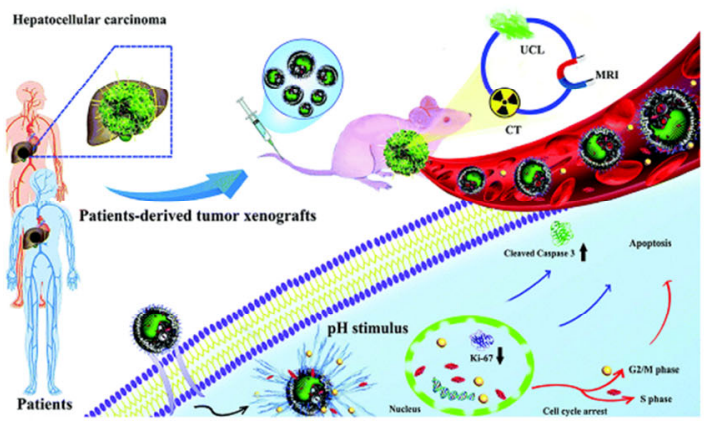

Figure 6 (a) Schematic presentation for dual-control drug release systems by using the dual-compartment mesoporous Janus nanocomposites (reproduced with permission from Ref. [78], ๑ American Chemical Society 2014). (b) Schematic illustration of the synthesis, modification and application of the Yin Yang-like OA-UCNPs@HPS NPs (reproduced with permission from Ref. [82], @ The Royal Society of Chemistry 2019). 
concentration gradient [87]. This method can reduce the damage of drug during synthesis. Considering some drugs are loaded on the surface, the burst release process should be controlled when it is applied. Moreover, some efforts are still necessary to enhance the encapsulation efficiency.

\subsection{Interaction}

The loading efficiency and the following drug release are strongly influenced by the interaction. The loading mechanism of drugs on carriers can be categorized into physical adsorption (molecular interaction) and chemical bonding (Fig. 7).

\subsubsection{Physical adsorption}

Weak molecular interaction mainly consists of van der Waals forces, hydrogen bonding, hydrophobic interaction, etc.

Considering the understanding in the interaction forces between carriers and drugs is helpful in optimizing the loading strategy, here we discuss the various interaction forces in this section. For hollow liposomes, hydrophobic interactions play an important role. Water-soluble drugs are stored in the hollow cavity, while hydrophobic drugs are stored in the phospholipid bilayers. Besides, Cationic lipids have good affinity with negatively charged cells, and therefore the drug molecules can be adsorbed on the carrier by electrostatic interaction [88]. $\mathrm{Xu}$ et al. utilized the interaction to bind negatively charged DNA chains to cationic liposomes, and release them in cells [89]. Apart from liposomes, electrostatic interactions also exist between drugs and other carriers with opposite charges. The positively charged DOX molecules could form a strong adsorption with the negatively charged surface of mesoporous silica, for instance $[25,37]$.

Moreover, $\pi-\pi$ stacking refers to a weak interaction that often occurs between aromatic rings. Some aromatic compounds, such as doxorubicin, doxycycline, mitoxantrone are likely to anchor to the carbon-based inorganic drug carriers $[49,90]$, or covalent organic frameworks (COFs) [91]. Chen et al. found that DOX was loaded onto multi-walled carbon nanotubes via $\pi-\pi$ stacking and then released under acidic condition by destroying the interaction [90].

\subsubsection{Chemical bonding}

Chemically combined drugs on carrier materials can be obtained by constructing amide, hydrazone bond, and disulfide bond between drug molecules and carriers [92]. Su et al. synthesized docetaxel-loaded polymeric complex micelles via two hydrazone linkages in the backbone, and the $\mathrm{pH}$-responsive drug release improved the therapeutic efficiency in cancer treatments [93].

In a word, interaction determines the stability of the action of drugs and carriers, thereby affecting its series of dynamic (a)

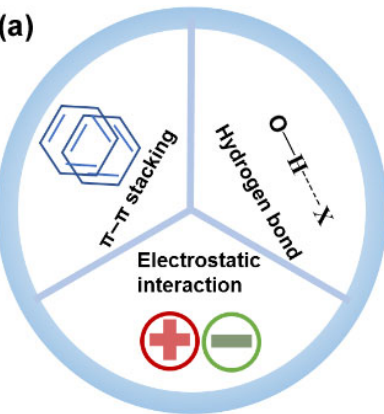

(b)

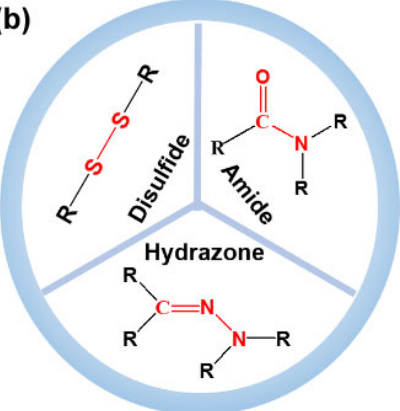

Figure 7 Illustration of the interaction between drugs and carriers. (a) physical interactions, such as $\pi-\pi$ stacking, hydrogen bond, and electrostatic interaction. (b) chemical bonds, such as disulfide bond, amide, and hydrazone bond. behaviors. Considering HoMS can provide multiple interfaces and spaces for drug loading, there are alternated physical and chemical interactions during the drug loading, and provide promising properties in drug release.

\section{Recognition}

In a smart targeted drug delivery system, recognition is the first step in the drug delivery process, and it is also an important function of the drug delivery system. With a targeted carrier being designed reasonably, it can effectively deliver the drug to the targeted site aiming to achieve the specific targeted delivery, as well as reducing the toxic side effects of the drug and distribution of the drug in normal tissues, which improves the utilization of drugs.

In contrast to solid nanoparticles, a hollow structure is composed of a shell and cavity, and the two parts can independently carry the functions of target recognition and drug storage. The outer shell is modified with an aptamer to realize the targeting; while the internal cavity is utilized as a drug reservoir and is not interfered upon external modification to ensure the activity of chemical molecules. Because of the targeting, the non-specific uptake of drugs in this site is reduced. Meanwhile, since the drugs are stored in the internal cavity of the hollow structures, the barrier of the outer shell is beneficial in further reducing the leakage of drugs [94]. Also, the outer shell layer interacts with biological components such as proteins on the cells through modification, which can determine the biodistribution and pharmacokinetic behavior of the drugcarrying system in vivo, avoiding a large amount of uptake by the reticuloendothelial system (RES) [95].

According to the type of mechanism, targeting is usually divided into passive targeting [96] and active targeting [97] (Figs. 8(a) and 8(b)).

\subsection{Passive targeting}

Passive targeting refers to taking advantages of the physiological structural characteristics of tissues and organs to make the carrier produce natural distribution differences in vivo to achieve targeting. Passive targeting mostly depends on the size effect of the drug or its carrier. For example, when intravenously injection is adopted, particles larger than $7 \mu \mathrm{m}$ are usually trapped by small capillaries in the lungs through mechanical filtration; particles larger than $200 \mathrm{~nm}$ are easily consumed by the reticuloendothelial system of the spleen and liver [98]. Therefore, a drug carrier with an appropriate size can be prepared according to the retention characteristics of different target organs for particles of different sizes.

In addition to the particle size, the properties of the particle surface, such as its electronegativity, hydrophobicity, and surface tension, also affect the distribution of drugs. For example, particles with a negative surface charge are easily taken up by the liver, while particles with a positive surface charge are easily taken up by the lungs [99].

\subsection{Active targeting}

Active targeting mainly refers to giving the drug or its carrier the ability to actively bind to the target, such as by coupling a probe molecule that can specifically bind to the target molecule on the cells; or applying an external field in vitro $[100,101]$.

Supramolecular action is a promising method in targeting system, which is mainly based on non-covalent bonds, such as hydrogen bonds, electrostatic interactions, $\pi-\pi$ interactions, van der Waals interactions $[102,103]$. The supramolecular nanovalve switch with adjustable host-guest function can realize the opening of specific lesions, ensuring the targeted delivery 


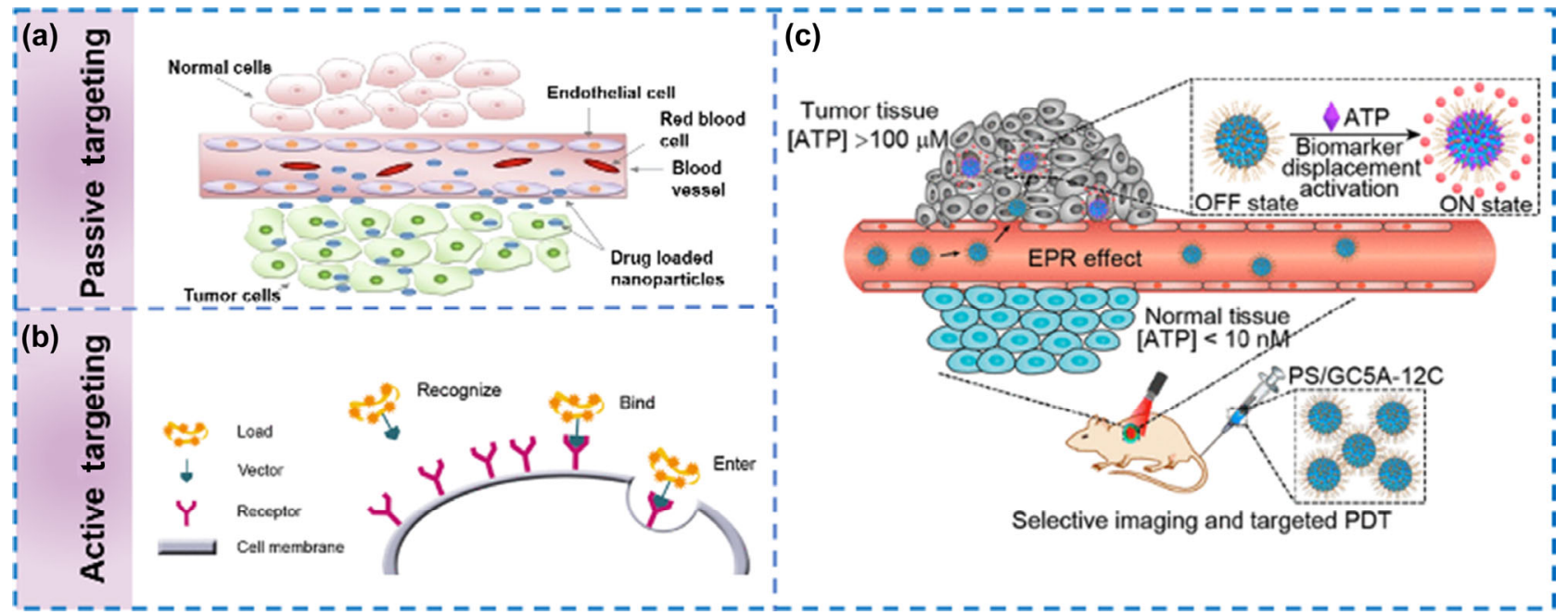

Figure 8 Schematic diagram of (a) passive targeting (reproduced with permission from Ref. [96], ( ) Springer Nature Switzerland AG 2019), (b) active targeting (reproduced with permission from Ref. [97], ( American Chemical Society 2019), and (c) the combination of passive targeting and active targeting (reproduced with permission from Ref. [105], @ American Chemical Society 2018).

of drugs, and provide the possibility for targeted, reversible and controllable treatment $[104,105]$. For example, Guo and co-workers constructed a guanidinium-modified calix[5] arene pentadodecyl ether (GC5A-12C) [105], and the photosensitizer (PS) was strongly bonded into it through the noncovalent host-guest interaction. When the carrier encountered a biomarker overexpressed in tumor tissues, the biomarker displaced PS, leading to the release of PS (Fig. 8(c)).

Another commonly used strategy is to modify the ligand on the surface of the carriers, such as antibody, peptide, protein, and small molecules [106]. When the carriers bring the drug into the tissue, the carriers can accumulate on the cell surface in large quantities and cause cell internalization relying on the specific recognition effect between the receptor and the ligand [91, 95, 107-109].

In addition to tumor cell targeting, the antibacterial agentloaded carrier can also be modified to construct a bacterialtargeted drug delivery system. It is able to use peptidoglycan and other glycolipids on the bacterial cell wall to modify the corresponding ligand on the surface of the carrier, such as $\mathrm{N}$-(2-aminoethyl)-3-aminopropyltrimethoxy-silane (DAMO), poly(propyleneimine) third-generation dendrimer (G3), trehalose. It can specifically recognize bacteria but not human host cells. For example, Yan and co-workers constructed a hollow oblate mesoporous silica (HOMSNs) as a drug delivery system for antimicrobial activities against mycobacteria [110]. Trehalose was used as the targeting ligand and isoniazid as the antibacterial agent. The exhibited enhanced antibacterial activity in vitro contributed to the increased interactions between HOMSNs and mycobacteria in the presence of trehalose. Similarly, some molecules can act as targeting agents for Gram-positive bacteria. Gao and co-workers designed a biodegradable supramolecular material based on cationic polyasparagine derivatives and column[5] aromatic hydrocarbons (CP[5]A) [111]. In order to improve the biocompatibility of Q-PAsp (BDA), CP[5]A was introduced into the polymer antibacterial agent through the supramolecular host-guest method, thereby obtaining a selective targeting of Gram-positive bacteria, and reducing the resistance of antibacterial drugs. Considering heterogeneous HoMS can provide different interfaces for molecule grafting, the multiple active targeting can be realized with heterogeneous HoMS. Then different shells can be targeted to different organelle and increase the curative effect [71].

Another strategy is to apply an appropriate force in vitro.
For example, a magnetic substance is introduced into the carrier matrix, under the effect of an external magnetic field, and then the pharmaceutical carrier may be aggregated with blood circulation to tumor tissues. Yue et al. [112] fabricated an iron oxide nanotube for the magnetically targeted paclitaxel (PTX) delivery. Under a condition of external magnetic field, the amount of PTX absorbed by cells increased significantly, and the killing effect of PTX on tumor cells was also improved.

In many studies, active targeting and passive targeting functions work together. For example, in tumor treatment, the passive targeting of enhanced permeability and retention effect (EPR) in mouses is used to enrich the drug-loaded particles at the tumor site, and then the modified ligand is utilized to achieve precise release of the drug in the targeted portion [113-115]. Jiang et al. developed a hyaluronic acid (HA)-coated nanogel core-shell structure as a drug carrier, which can be enriched in tumor tissues under the combined effect of the EPR effect and HA active targeting [116].

\section{Response}

Stimulus-responsive release occurs when certain conditions are triggered, which is consisted of endogenous stimulus and exogenous stimulus. Research on the response of drug delivery systems to endogenous stimulus mainly focuses on the tumor microenvironment (TME), including $\mathrm{pH}$, enzyme, and glutathione (GSH). In addition, the response to the exogenous stimulus also has practical application significance, such as the responsive release to temperature and light.

In terms of the design of a smart responsive drug carrier, there are two aspects to be considered. The first strategy is using materials that are sensitive to $\mathrm{pH}$, enzyme, temperature, and other stimuli. The other is through the functionalization of materials, such as the construction of the interaction between host and guest, which can respond to a specific stimulus, and thus exhibit different release properties (Fig. 9).

\subsection{Endogenous stimulus}

The endogenous stimulus response mainly refers to the response to the tumor microenvironment. The TME is mainly composed of tumor cells and surrounding tissue cells. The acidic environment in the tumor microenvironment, enzymes, GSH in the cytoplasm and nucleus, etc. can all be used as endogenous stimuli to enable the responsive release of drugs in tumor 


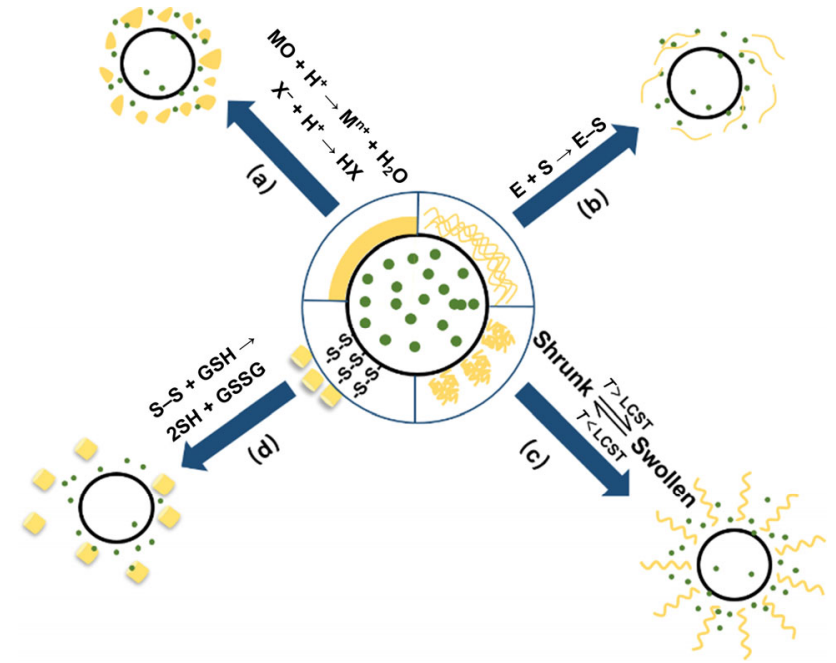

Figure 9 Schematic diagram of stimulus-response. (a) In acid-response, the carrier undergoes degradation or protonation when encountering acid (b) In enzyme-response, enzymes and substrates binding lead to collapse of carrier structure (c) In temperature-response, the polymer carrier undergoes a phase transition. Below LCST, the material swells; above LCST, the material shrinks. (d) In reduction-response, the disulfide bond of the material is reduced by glutathione, and the structure is destroyed.

tissues or cells, so as to improve the tissue distribution of drugs, and reduce their side effects of drugs.

\subsubsection{Response to $\mathrm{pH}$}

$\mathrm{pH}$-responsive nanocarriers can be used for the controlled release of drugs in the weak acid microenvironment of tumors and play an active role in biomedical applications such as targeted release of drugs and targeted therapy of cancer.

Developing an acid-labile nanocarrier is one of the strategies to construct $\mathrm{pH}$-responsive drug delivery [117-120]. For example, polypropylene, $\mathrm{CaCO}_{3}$ [121], $\mathrm{ZnO}$ [122], $\mathrm{MnO}_{2}$ [123], MOFs [40] can swell or degrade in an acidic environment, resulting in drug release at the TME. Ke et al. designed a poly $(d, 1-$ lactic-co-glycolic acid) (PLGA) hollow microsphere containing $\mathrm{NaHCO}_{3}$ [57]. The anti-cancer drug DOX was encapsulated through physical adsorption to avoid a change in the drug activity. Once entered into an acidic environment, the $\mathrm{CO}_{2}$ bubbles generated by $\mathrm{NaHCO}_{3}$ punched holes in the PLGA shell to release the drug.

This work changed the driving force through the design of chemical reactions, combined polymer degradation and drug diffusion, and provided a platform for the design of polymerbased drug carriers (Fig. 10(a)). Besides, hydroxyapatite (HAp) materials dissolve when the $\mathrm{pH}$ value is smaller than 5, exhibiting acid sensitivity. Weerasuriya and co-workers [124] enclosed anticancer drugs in hollow hydroxyapatite nano-carriers and they found that HAp was completely dissolved at $\mathrm{pH}$ 5, which facilitates targeted delivery to tumor tissue.

The realization of a $\mathrm{pH}$-responsive carrier can also be achieved by constructing materials that undergo protonation under acidic conditions. Examples include weakly basic polymers with basic groups such as amino, pyridine, and imidazole groups, or weakly acidic polymers with carboxylic acid groups [125]. These functional groups react as follows under acidic conditions:

$$
\mathrm{X}^{-}+\mathrm{H}^{+} \rightarrow \mathrm{HX}
$$

The charge and structure change after the group is protonated, leading to a change in the configuration of the polymer, which triggers the response. As shown in Fig. 10(b), Wang et al. developed a unique composite which coated $\mathrm{AuNC} / \mathrm{Fe}(\mathrm{OH})_{3}$ with PAA to realize a $\mathrm{pH}$-responsive release [126]. PAA can be protonated under acidic conditions, which weakens the electrostatic force between drugs and carriers, inducing the drug release.

\subsubsection{Response to glutathione}

High concentrations of glutathione in the tumor environment can trigger a redox-responsive release by cleaving the disulfide bond, which is between the carrier and the drug molecule.

In response to reduced glutathione, the following reaction occurs:

$$
\mathrm{S}-\mathrm{S}+\mathrm{GSH} \rightarrow 2 \mathrm{SH}+\mathrm{GSSG}
$$

GSH acts as a reducing agent to break the disulfide bond (SS), generating oxidized glutathione (GSSG) by itself, and reducing the disulfide bond to thiol group $(-\mathrm{SH})$.

A redox-responsive drug delivery system can be obtained through the construct of a disulfide bond [127]. Generally, the conjugate is coated on the surface of the carrier, or used as a gatekeeper to lock the pores. Commonly used disulfide bondforming conjugates include cyclodextrin (CD) [128-130], poly(b-amino esters) (PAE) [131], chitosan (CS) [132], and transferrin [133]. Silicon particles are commonly used framework materials for preparing responsive carriers. $\mathrm{Lu}$ et al. [128] developed a hollow mesoporous organosilica nanoparticle, and $\alpha$-cylodextrin $(\alpha$-CD) was chosen as the capping molecule. The DOX loading capacity reached to $1,060 \mathrm{mg} \cdot \mathrm{g}^{-1}$ owing to the hollow cavity, and the redox-responsive release was observed owing to the breaking of the S-S in the thioether group (Fig. 11(a)).

Shi and co-workers constructed a redox-responsive gene release by the decoration of PAE via a disulfide linker on the large pore-sized hollow mesoporous organosilica [131], which was applied to co-deliver siRNA and DOX. Antitumor effect exhibits the siRNA/DOX@HMONs-ss-PAE group has the highest antitumor activity with only $7.61 \%$ of tumor volume, owing to the controlled co-delivery of siRNA and DOX in the tumor microenvironment with high GSH concentration (Fig. 11(b)).

GSH-response improved the release amount and treatment efficiency of anti-tumor drugs. Moghaddam et al. [134] reported a redox-responsive release of DOX from a hollow mesoporous sphere. As a result, $60 \%$ of DOX was released in two weeks with the existence of $10 \mathrm{mM} \mathrm{GSH}$, while only $14 \%$ was released without GSH. In addition to a single GSH response, a triple-responsive ( $\mathrm{pH}$, reduction, and light) hollow mesoporous silica nanocarrier fabricated by Zhao et al. [135] indicated greater potential for controlled release of drugs and disease treatment in complex pathological environments.

\subsubsection{Response to enzymes}

Enzyme substrate fragments are coupled to the host material through covalent bonds, hydrophobic interactions or electrostatic interactions to give the nanocarrier enzyme-sensitive properties. The expression levels of some kinds of enzymes are related to the occurrence and development of tumors, such as metalloprotease, cathepsin B, hyaluronidase, secretory phospholipase, alkaline phosphatase, prostate antigen, oxidoreductase, $\alpha$-amylase and $\gamma$-glutamyl transferase. The release of the drug depends on the specific binding of the enzyme (E) to the substrate (S):

$$
\mathrm{E}+\mathrm{S} \rightarrow \mathrm{E}-\mathrm{S}
$$

Surface functionalized hollow mesoporous silica was often used as an enzyme-induced drug carrier. As shown in Fig. 12(a), Zhu and co-workers combined the hollow mesoporous silica with enzyme-degradable poly(L-lysine) to construct an enzyme- 
(a)
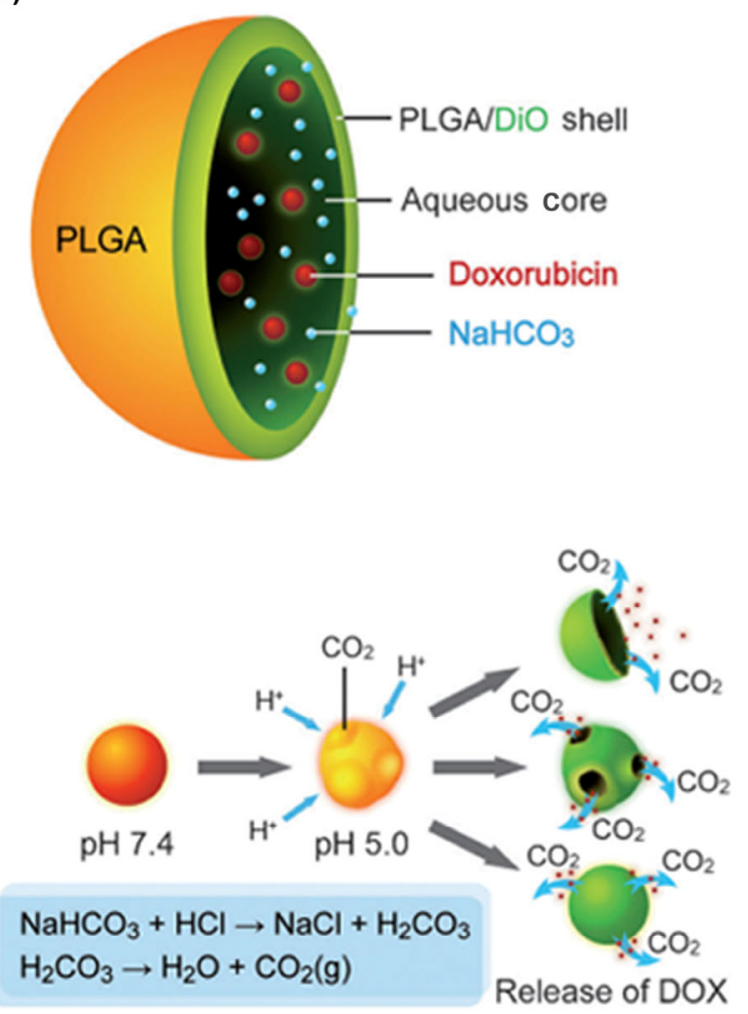
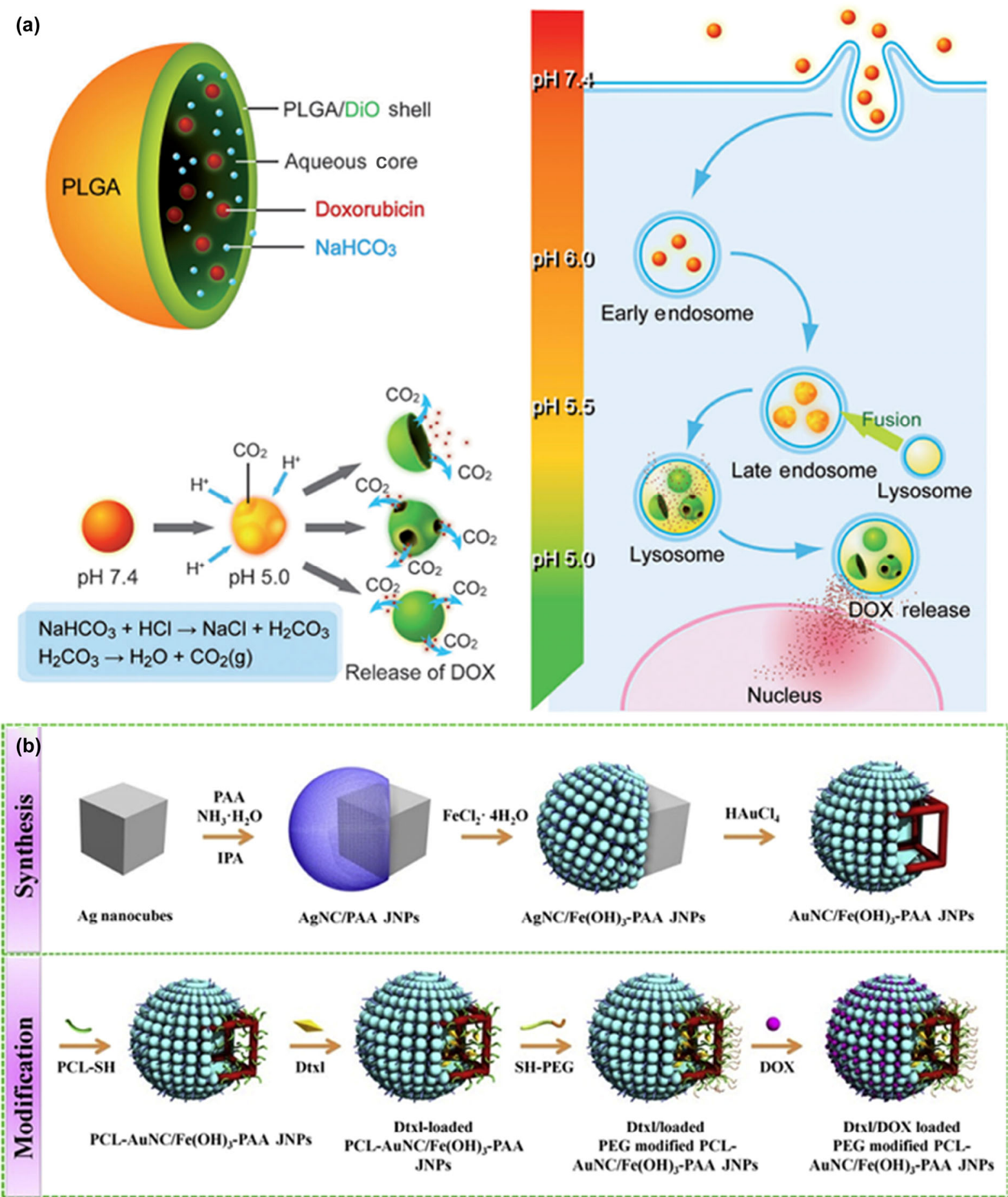

Figure 10 (a) Schematic illustration of the structure of a PLGA hollow microsphere containing doxorubicin and the mechanism of drug release (left), as well as the intracellular trafficking and release of the drug from the $\mathrm{pH}$-responsive microspheres (right) (reproduced with permission from Ref. [57], (c) WILEY-VCH Verlag 2011). (b) 1. Schematic illustration of the fabrication, modification and application of AuNC/Fe(OH) 3 - PAA Janus NPs (reproduced with permission from Ref. [126], (C) Elsevier B.V. 2018).

triggered drug and gene co-delivery system. The in vitro release behavior indicated that the release rate of fluorescein and cytosine-phosphodiester-guanine oligodeoxynucleotide can be controlled by changing the concentration of the enzyme [136].

In the TME, the expression and activity of enzymes are significantly different from the outside. Using an enzyme responding to control drug release can improve therapeutic efficiency and reduce damage to the human body. $\alpha-C D$ can be enzymatically hydrolyzed by $a$-amylase, which consequently is used as a gatekeeper of enzyme-responsive drug carriers [137].
Another example is modifying the hollow carbon nitride nanosphere (GHCNS) with polysaccharide hyaluronic acid, and then using it as a drug carrier for photochemical internalization. HA can be degraded into fragments by intracellular lysosomal hyaluronidase (Hyal) to enable cargo release, thereby achieving an enzyme-triggered drug release (Fig. 12(b)) [138].

\subsection{Exogenous stimulus}

In addition to the endogenous stimuli brought by the TME, external environmental stimuli including light and temperature can also coordinate tumor therapy. 
(a)
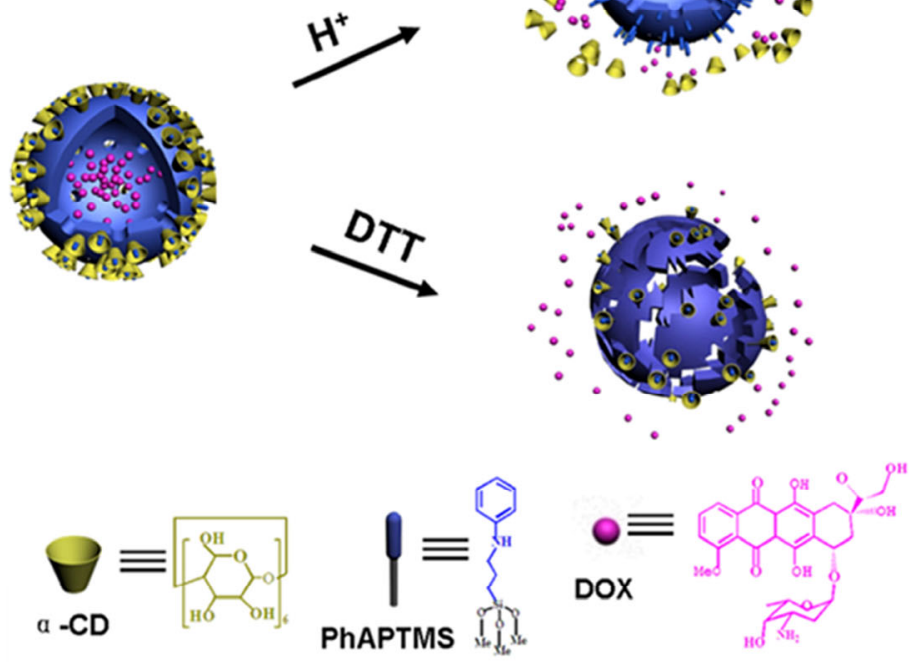

(b)

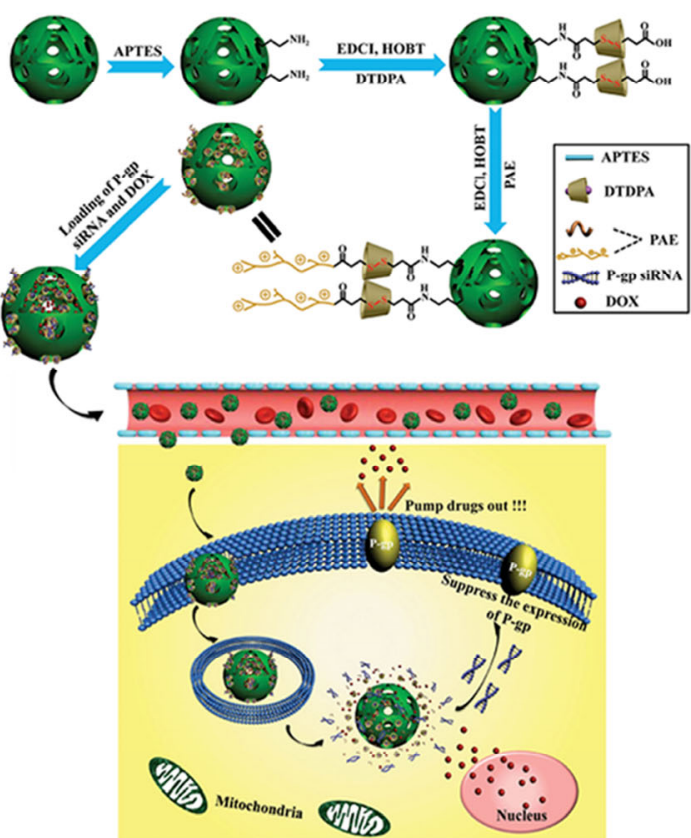

Figure 11 (a) Schematic illustration of Pore channels and framework dual responsive, controlled release of DOX from DOX@HMONs/CD (reproduced with permission from Ref. [128], ( $\odot$ Elsevier B.V. 2019). (b) Construction of P-gp modulator siRNA and DOX co-loaded HMONs-ss-PAE system, and c) its effects on suppressing P-gp expression for the treatment of MDR (reproduced with permission from Ref. [131], () WILEY-VCH Verlag 2016).

(a)

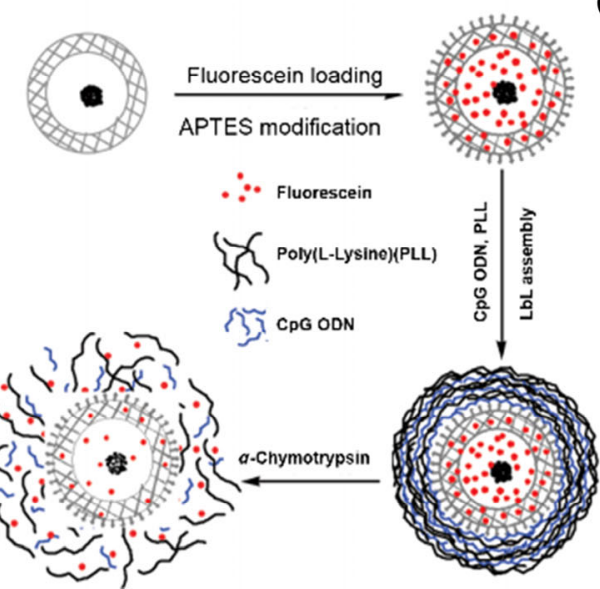

(b)

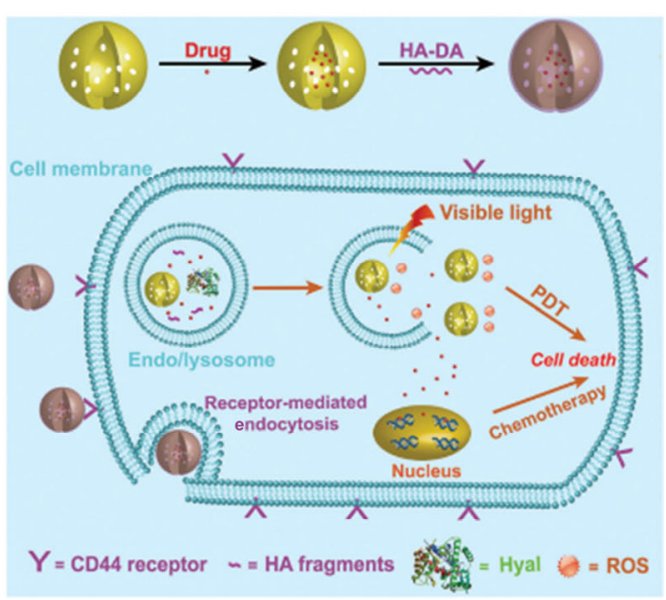

Figure 12 (a) Schematic procedure for preparation of the fluorescein and CpG ODN-loaded HMS/PLL particles and enzyme-triggered release (reproduced with permission from Ref. [136], @ American Chemical Society 2011). (b) Schematic illustration of a target and stimuli-responsive platform based on drug loaded GHCNS-HA nanoparticles for the PCI mediated endo/lysosome escape and synergistic therapy (reproduced with permission from Ref. [138], ( $)$ The Royal Society of Chemistry 2016).

\subsubsection{Response to near-infrared (NIR)}

According to different wavelengths of the light, the response should be part to ultraviolet (UV), visible, or NIR. Among them, near-infrared light is easier to penetrate the skin and tissues, then reach the tumor. Therefore, NIR light-responsive nanomaterials possess potential for applications in nanomedicine and phototherapy.

Carbon [139, 140], gold [141], and upconversion nanoparticles (UCNPs) [142] were often utilized in NIR responsive drug carriers owing to their NIR absorption capacity. Lee and co-workers [139] fabricated the hollow mesoporous silica/carbon nanoparticles as a DOX carrier. With efficient NIR photothermal conversion and high DOX loading capacity, the polyethylene glycol-Si/C-DOX NPs are demonstrated to have remarkable cancer-cell-killing ability via combinational chemothermal therapy (Fig. 13(a)). UCNPs can convert NIR light into different wavelengths of light in UV and visible regions and can avoid related damage to the tissue from direct UV irradiation [143]. A yolk-shell NaYF4:Yb,Tm@NaLuF 4 UCNPs was synthesized by Li et al. [144] and was then used as a carrier for anti-cancer drug chlorambucil. A high loading capacity of $49 \%$ was realized, along with a high photolysis efficiency of $88 \%$, and the drug can only be triggered by photolysis without pre-released in living animal tissues (Fig. 13(b)).

\subsubsection{Response to temperature}

Temperature-sensitive drug carriers are commonly found in temperature-sensitive hydrogels, such as poly( $\mathrm{N}$-isopropyl acrylamide) (PNIPAM) and its complexes [145-149]. This type of hydrogel has a certain proportion of hydrophilic and hydrophobic groups. A change in temperature can affect the intramolecular and intermolecular interactions of these groups with water, thereby changing the network structure of the hydrogel. 


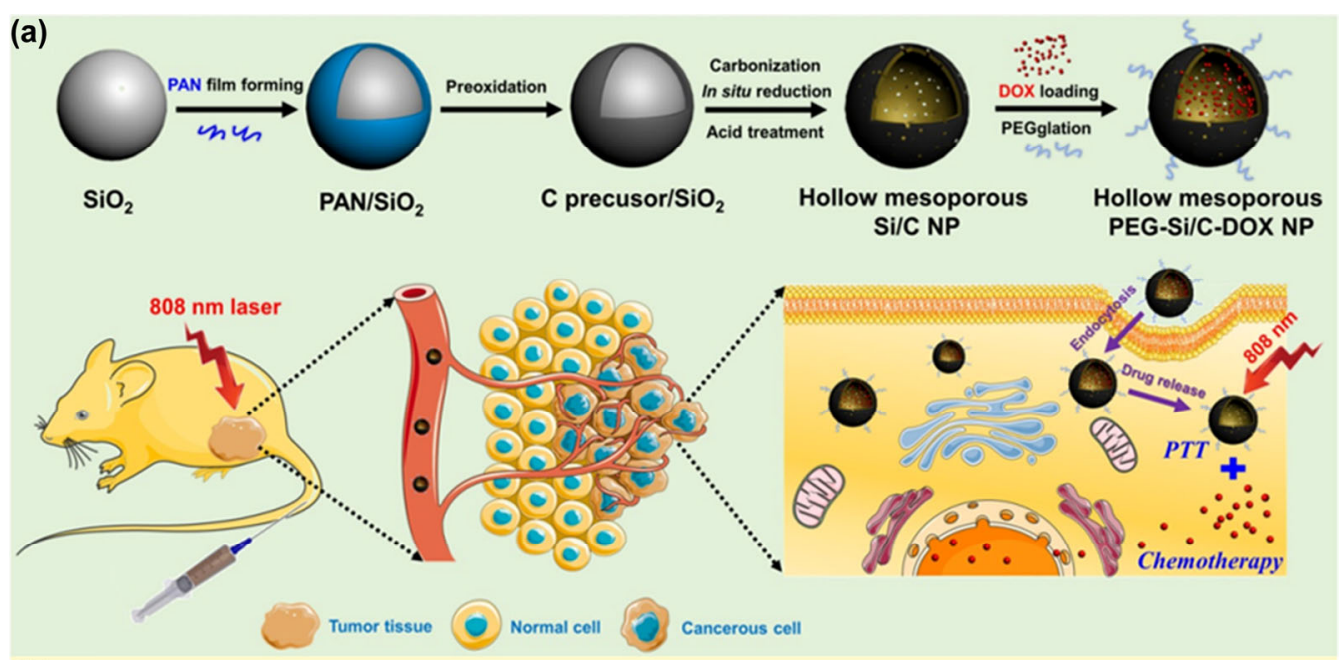

(b)
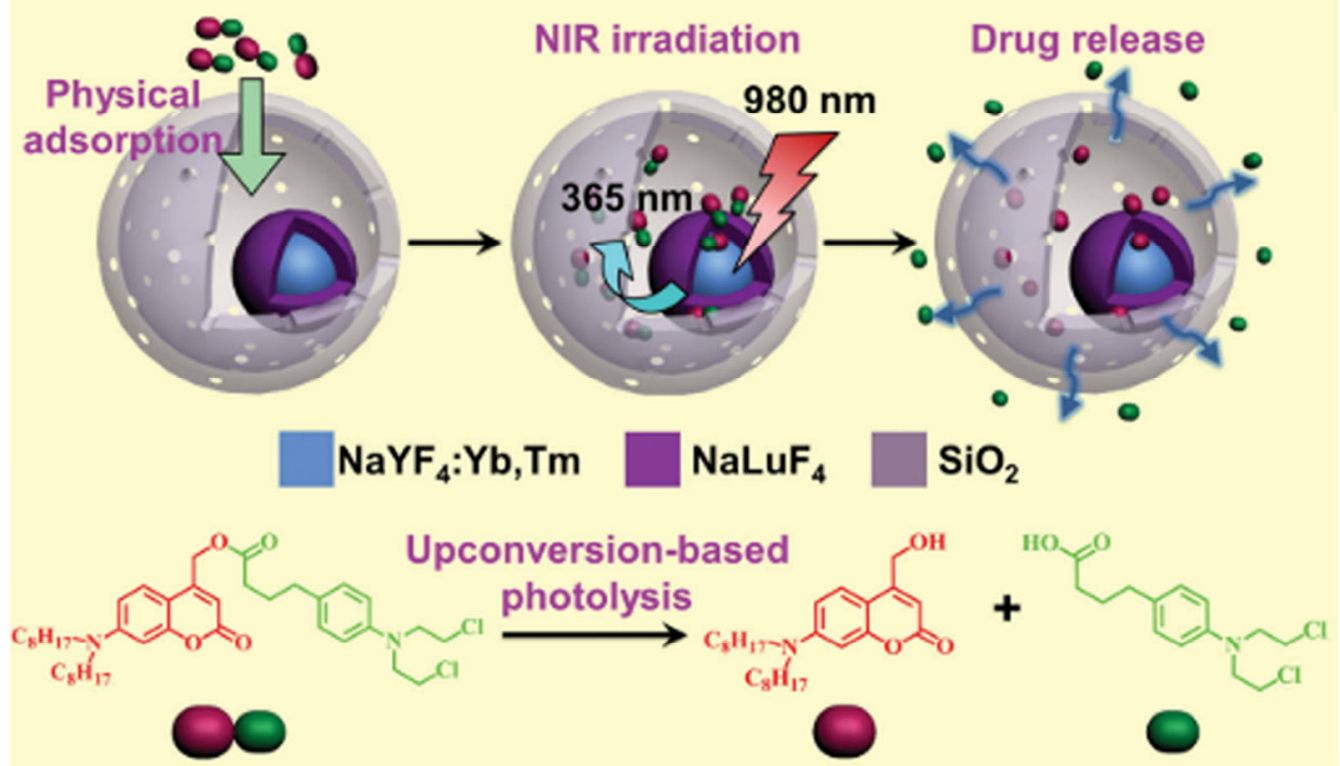

Figure 13 (a) Schematic illustration of fabrication of hollow mesoporous PEG-Si/C-DOX NP and b) its application for photoacoustic imaging-guided chemo-thermal therapy (reproduced with permission from Ref. [139], () Ivyspring International Publisher 2017). (b) Schematic illustration of the NIR-regulated upconversion-based PDD and the photolysis of the prodrug under upconversion emission from the YSUCNPs (reproduced with permission from Ref. [144], () WILEY-VCH Verlag 2013).

Regarding the source of heat, in addition to photo-thermal, magnetothermal also matters. Song et al. used mPEG-b-PHEP micelles as nano-carriers, encapsulating magnetic nanocubes and tumor-killing drug emodin, to achieve magnetic resonance imaging (MRI)-guided combined treatment of malignant tumors with magnetic resonance imaging [150]. Under magnetothermal stimulus, the chemotherapeutic drug emodin can be rapidly released from the viscous PHEP core of micelles, and its release rate is significantly better than that of traditional micelles with polylactic acid as the core, which improves the treatment efficiency significantly.

Zhao et al. developed a smart nanocarrier, where the hollow gold nanosphere was used as the drug reservoir as well as photothermal conversion agent, and the surface was modified with a temperature-sensitive polymer and AS1411 aptamer, so as to achieve light and heat-oriented cell uptake and treatment [149]. The polymer undergoes a phase transition at lower critical solution temperature (LCST), from a swollen hydrated state to a shrunken hydrophobic state, allowing cell entry in a controlled manner.

Aside from light and temperature, factors such as $\mathrm{pH}$, humidity, and bacterial concentration in the ecological environment will also bring about the responsive release of guests [151]. And the HoMS system can be endowed with multiple responses no matter the endogenous or the exogenous stimulus, thus bringing a bright future for smart drug release.

\section{Release}

With progress in pharmaceutical technology, other sophisticated controlled-release platforms are being developed. Carriers with controlled delivery of drugs can overcome problems caused by conventional free drugs, including poor solubility, limited stability, rapid clearing, and in particular, lack of selectivity. Hollow material has become an important research direction over past decades owing to their great application potentials in fields like bio-imaging, controlled release, tumor therapy, and tissue engineering.

\subsection{Single mode release}

Release kinetics are divided into zero order release, first order release, the Higuchi model, etc. [152-154]. Zero-order release is 
a constant-rate release, usually observed in oral, transdermal drug delivery [155]. The first-order means that the release concentration is proportional to the release rate, and it is suitable for the case where the drug is evenly distributed in the carrier. The Higuchi model is that the release amount is proportional to the $1 / 2$ power of time, which is suitable for the case where the drug is unevenly distributed on the carrier.

According to the plasma concentration, drugs can be classified into ordinary preparation, sustained-release drugs, and controlled-release drugs [156]. Ordinary preparation is a rapid decrease in blood drug concentration, which requires multiple administrations; the sustained release drug is a preparation that slowly releases the drug at a non-constant rate, and the drug concentration decreases slowly with time. Controlled release drug refers to the release of the drugs regularly and quantitatively, keeping the plasma drug concentration within a constant range.

\subsection{Multi-level release}

In the aspect of plasma concentration control and ecological management, it is necessary for drug concentration to be environmentally adaptable, that is, to be able to adjust the dose as needed. As a result, multi-level release plays an important role. Multi-level release refers to that the drug delivery through carriers that can be divided into multiple stages $(\geq 2)$ with different rates. The driving force of multi-stage release is the concentration gradient that is jointly affected by physical barriers and chemical diffusion, no matter the continuous or the stimulus response release stage.

Lu and co-workers [157] constructed the yolk-shell nanoparticles as a multi-stage release platform for ibuprofen. In the first $1 \mathrm{~h}$, drug molecules absorbed on the surface were quickly released, and reached to the plateau in the following $5 \mathrm{~h}$. In the second stage, the release reached another plateau and releasing $63 \%$ of loaded drugs. In the third stage, molecules absorbed in the pores were released more slowly, and finally arrived at $75 \%$.

The complex environment puts forward higher requirements for the adaptability of drug carriers. During the sterilization, an ideal antibacterial system needs to meet the following requirements: (1) the rapid release of antibiotics to the environment at the concentration required for bacteriostatic treatment or sterilization; (2) maintenance of this concentration for a long time to prevent bacterial growth; (3) autodetection of foreign pathogens and self-responsive release of the reserved antibiotics.

Zhao et al. [76] used hollow multi-shelled $\mathrm{TiO}_{2}$ as a drug carrier and antibacterial agent methylisothiazolinone (MIT) as a model drug. MIT molecules adsorbed in the outer surface can be discharged quickly as the weaker driving force required, thus bringing out the bactericidal concentration. Subsequently, the $\pi-\pi$ stacked drug molecules in the HoMS' cavity slowly desorb and diffuse into the environment, resulting in long-term sustained release, which is conducive to bioclean surroundings for a long period of time. More importantly, when pathogens are added into the environments, HoMSs can release the stored drugs timely, thereby killing the bacteria. The difference in the adsorption characteristics of different spatial parts of HoMSs and the multi-level physical barrier effects of the shells, make the molecules in different adsorption states and adsorption sites sequentially release at different time.

Self-decomposable materials are one type of carrier with multi-level performance. Li's group worked on the design of $\mathrm{SiO}_{2}$-based self-decomposable nanoparticles as effective drug carriers $[83,158,159]$. In these works, the release of drug molecules was accompanied with the carrier decomposition, forming hollow spheres gradually until $\mathrm{SiO}_{2}$ was completely degraded.

\subsection{Sequential release}

In more complex pathological situations, a combination of multiple drugs is required, and the delivery of different drugs has specific requirements regarding spatial and temporal ordering [160]. The design of sequential release carriers provides a solution to this problem.

Currently, there are three challenges in constructing a multidrug sequential release carrier: (1) The chemical reaction among multiple drugs needs to be avoided when they are applied; (2) the drug loading capacity should satisfy the required amount of various drugs; (3) the release order of different drugs needs to be controlled. In order to address these issues, the current research mainly focuses on establishing multi-space drug carriers, separating different drugs in different physical spaces; adopting different loading methods and different adsorption modes according to the characteristics of the carriers and drugs; applying different release driving forces to programmatically control the sequential release of drugs.

Hollow structures provide a platform for sequential drug release. A direct approach to realize the sequential release is loading different drugs into distinct spatial positions of the carrier. In the absence of external environmental stimuli, the concentration gradient as the only driving force will bring a temporal-staggered release, and the release rates of the two guest molecules are different, although the overlap releasing of drugs is inevitable sometimes [161-164]. Dou et al. obtained an O-carboxymethyl chitosan microspheres (O-CMCS) hydroxyapatite collagen scaffold [164]. Two kinds of growth factor related to bone regeneration, bone morphogenetic protein-2 (BMP-2) and vascular endothelial growth factor (VEGF), were loaded into O-CMCS and hydroxyapatite collagen, respectively. The release behavior of these two different loads was studied. Results presented that the VEGF stored externally burst out significantly, reaching $75.45 \%$ in the first day, while $16.2 \%$ of the BMP- 2 molecules stored internally were released over the same duration.

If different stimuli are applied to the two parts, the difference in spatial position combined with the response to the environmental stimulus will work together to drive a more controllable sequential release $[165,166]$. For example, Cong et al. synthesized a hybrid dual-responsive microcontainer, in which the $\mathrm{pH}$-responsive polymer microspheres were loaded with 5(6)carboxyfluorescein diacetate (CFDA) [165]. Then stellate mesopore silica nanoparticles grafted with temperature-sensitive poly(N-isopropylacrylamide) (PNIPAM) were used as the carriers for Nile red, and they were modified on the exterior surfaces of CFDA contained polymer microspheres by Pickering emulsion method. When the temperature rose above the LCST temperature of PNIPAM, Nile red was released; while the $\mathrm{pH}$ dropped to 4, CFDA in the polymer microspheres were released (Fig. 14(a)). Similarly, Xu et al. constructed an organic core-shell structure of $\mathrm{PS}_{n}(\mathrm{P} 2 \mathrm{VP}-\mathrm{b}-\mathrm{PAA})_{n}$ copolymer@PNIPAM [166]. The inner core was loaded with hydrophilic Fluorescein isothiocyanate (FITC), and the outer shell was loaded with hydrophobic Nile red. By in situ loading, the co-loading of hydrophilic and hydrophobic drugs was realized. The multi-drug system also has the ability to respond to multiple stimuli. The configuration of the outer polymer chain was affected by temperature variations, thereby stimulating the release of the Nile red, while the lower $\mathrm{pH}$ can stimulus the release of FITC by modifying the structure of the polymer, so as to achieve programmatic and controllable sequential release (Fig. 14(b)). 

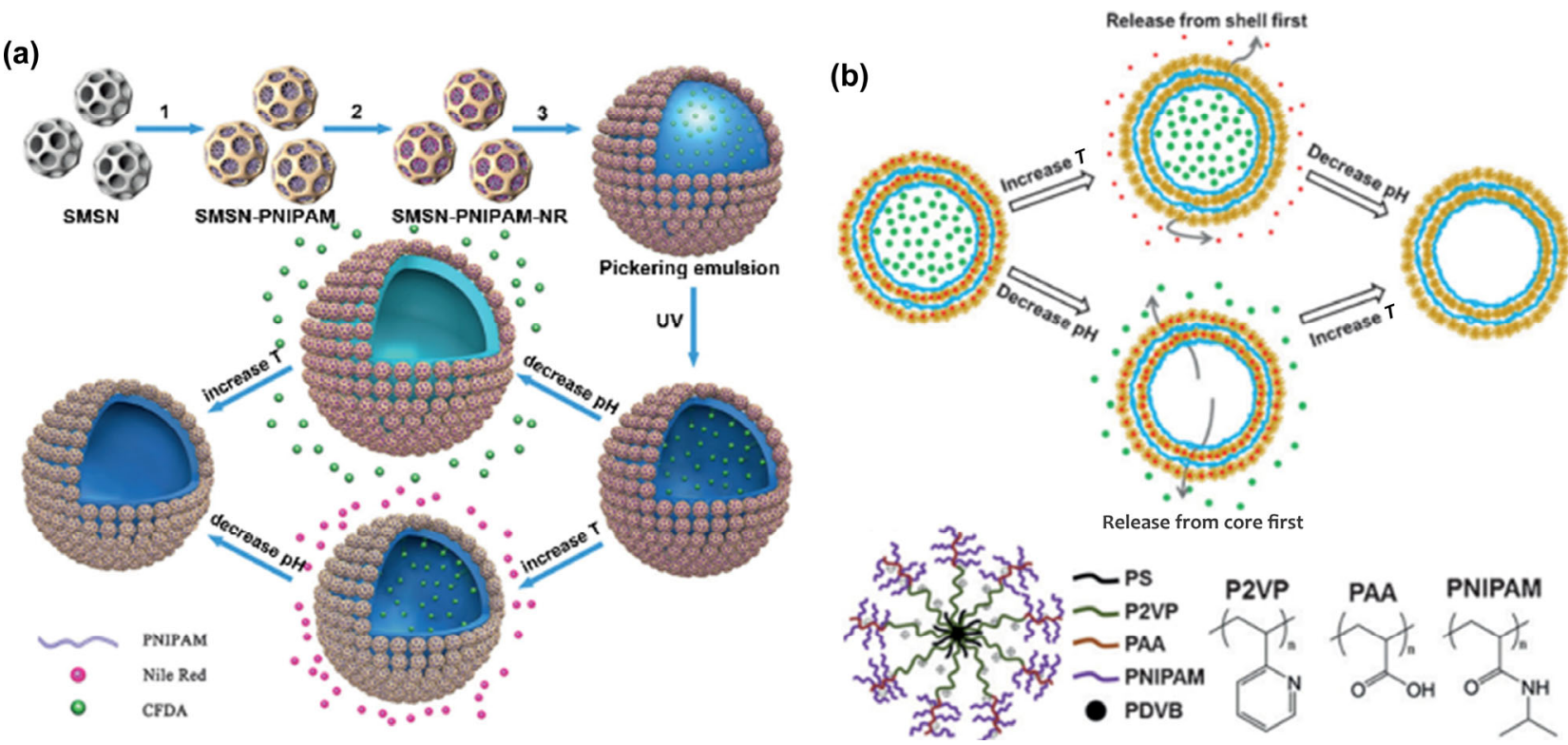

Figure 14 (a) Synthesis of microcapsules and pre-programmable release of two payloads from the microcapsules (reproduced with permission from Ref. [165], (C) The Korean Society of Industrial and Engineering Chemistry 2015). (b) The programmable encapsulation and release of two types of molecules from the microcapsule core and shell regions, and chemical structure of the SGQP (reproduced with permission from Ref. [166], ( ) WILEY-VCH Verlag 2013).

\section{Conclusion and prospect}

As participants in the drug delivery system, the hollow structures possess great advantages: Hollow cavities and shells contribute to large loading capacity; a tailored structure helps control the release rate; variable compounds adapt to flexible application; surface modification facilitates smart responsive release. This review has concluded the three processes of drug delivery system, which are recognition, response and release, determining by the driving force and the molecular interaction. Therefore, precise control on the carrier's structure is crucially important. Although many achievements have been made in related research, more efforts are still necessary in the material design for advanced drug delivery systems.

First of all, in terms of synthesis, precise synthesis for hollow structures with specific structures is necessary. Especially in case of multi-level drug carriers, the pores, internal cavity structure, and surface regulation need be precisely controlled. We can expect a heterogeneous HoMS with different interlayer compositions or HoMS with multi-cores can achieve multi-level and sequential drug delivery easily. For example, a heterogeneous HoMS can provide various interfaces and spaces, and then divide the drug molecules in the physical space. By adjusting the dynamic induced by a physical barrier and chemical interaction caused by multiple shells, the load and sequential release of multiple drugs can be achieved, which has great potential for application in combination therapy. Furthermore, if solid particles can be assembled into hollow structures when entering the body, they will greatly promote the development of modern medicine.

Secondly, research on smart and sequential release drug delivery systems is just started. We can expect the carriers can automatically find the target and release the drug at the corresponding location according to environmental factors such as $\mathrm{pH}$ difference and concentration difference, which are the goals of self-responsive and self-motive smart carriers. Considering HoMS has the great potential in sequential drug delivery, what we should take account into is how to reveal the unique property of HoMS by rational design to realize a better therapeutic effect. This is not only to improve patient tolerance clinically, but also to address the scientific issues of spatiotemporal ordering. These require researchers to have a more in-depth and precise understanding of the release mechanism and integrate the previous release principles. We are confident that hollow nanostructures will have expansion applications in drug delivery in the near future.

\section{Acknowledgements}

This work was financially supported by the National Natural Science Foundation of China (Nos. 21821005, 21820102002, and 21971244) and the National Key R\&D Program of China (No. 2016YFB0600903).

\section{References}

[1] Karimi, M.; Ghasemi, A.; Sahandi Zangabad, P.; Rahighi, R.; Moosavi Basri, S. M.; Mirshekari, H.; Amiri, M.; Shafaei Pishabad, Z.; Aslani, A.; Bozorgomid, M. et al. Smart micro/nanoparticles in stimulus-responsive drug/gene delivery systems. Chem. Soc. Rev. 2016, 45, 1457-1501.

[2] Cai, W.; Wang, J. Q.; Chu, C. C.; Chen, W.; Wu, C. S.; Liu, G. Metalorganic framework-based stimuli-responsive systems for drug delivery. Adv. Sci. 2019, 6, 1801526.

[3] Mura, S.; Nicolas, J.; Couvreur, P. Stimuli-responsive nanocarriers for drug delivery. Nat. Mater. 2013, 12, 991-1003.

[4] Wu, M. X.; Yang, Y. W. Metal-organic framework (MOF)-based drug/cargo delivery and cancer therapy. Adv. Mater. 2017, 29, 1606134.

[5] Sharma, H.; Kumar, K.; Choudhary, C.; Mishra, P. K.; Vaidya, B. Development and characterization of metal oxide nanoparticles for the delivery of anticancer drug. Artif. Cells, Nanomed. Biotechnol. 2016, 44, 672-679.

[6] Liu, Y. B.; Castro Bravo, K. M.; Liu, J. W. Targeted liposomal drug delivery: A nanoscience and biophysical perspective. Nanoscale Horiz. 2021, 6, 78-94.

[7] Yang, B.; Zhou, S.; Zeng, J.; Zhang, L. P.; Zhang, R. H.; Liang, K.; Xie, L.; Shao, B.; Song, S. L.; Huang, G. et al. Super-assembled core-shell mesoporous silica-metal-phenolic network nanoparticles for combinatorial photothermal therapy and chemotherapy. Nano Res. 2020, 13, 1013-1019.

[8] Wu, J.; Zhu, Y. J.; Cao, S. W.; Chen, F. Hierachically nanostructured mesoporous spheres of calcium silicate hydrate: Surfactant-free sonochemical synthesis and drug-delivery system with ultrahigh drug-loading capacity. Adv. Mater. 2010, 22, 749-753. 
[9] Folkman, J.; Long, D. M. The use of silicone rubber as a carrier for prolonged drug therapy. J. Sur. Res. 1964, 4, 139-142.

[10] Iwanaga, H.; Shibata, N. Growth mechanism of hollow $\mathrm{ZnO}$ crystals from ZnSe. J. Cryst. Growth 1974, 24-25, 357-361.

[11] Li, W.; Liu, J.; Zhao, D. Y. Mesoporous materials for energy conversion and storage devices. Nat. Rev. Mater. 2016, 1, 16023.

[12] Qu, H. Q.; Ma, Y. R.; Gou, Z. L.; Li, B.; Liu, Y. R.; Zhang, Z. X.; Wang, $\mathrm{L} . \mathrm{Ni}_{2} \mathrm{P} / \mathrm{C}$ nanosheets derived from oriented growth Ni-MOF on nickel foam for enhanced electrocatalytic hydrogen evolution. J. Colloid Interface Sci. 2020, 572, 83-90.

[13] Li, X. Y.; Rong, H. P.; Zhang, J. T.; Wang, D. S.; Li, Y. D. Modulating the local coordination environment of single-atom catalysts for enhanced catalytic performance. Nano Res. 2020, 13, 1842-1855.

[14] Zhang, J.; Karmakar, S.; Yu, M. H.; Mitter, N.; Zou, J.; Yu, C. Z. Synthesis of silica vesicles with controlled entrance size for high loading, sustained release, and cellular delivery of Therapeutical proteins. Small 2014, 10, 5068-5076.

[15] Peng, L.; Hung, C. T.; Wang, S. W.; Zhang, X. M.; Zhu, X. H.; Zhao, Z. W.; Wang, C. Y.; Tang, Y.; Li, W.; Zhao, D. Y. Versatile Nanoemulsion assembly approach to synthesize functional Mesoporous carbon nanospheres with tunable pore sizes and architectures. J. Am. Chem. Soc. 2019, 141, 7073-7080.

[16] Wang, W.; Tang, M. H.; Zheng, Z. Y.; Chen, S. L. Alkaline polymer membrane-based ultrathin, flexible, and high-performance solid-state Zn-Air Battery. Adv. Energy Mater. 2019, 9, 1803628.

[17] Li, N.; Zhang, Q.; Liu, J.; Joo, J.; Lee, A.; Gan, Y.; Yin, Y. D. Sol-gel coating of inorganic nanostructures with resorcinol-formaldehyde resin. Chem. Commun. 2013, 49, 5135-5137.

[18] Gao, M.; Zeng, J.; Liang, K.; Zhao, D. Y.; Kong, B. Interfacial assembly of mesoporous silica-based optical heterostructures for sensing applications. Adv. Funct. Mater. 2020, 30, 1906950.

[19] Slowing, I. I.; Trewyn, B. G.; Giri, S.; Lin, V. S. Y. Mesoporous silica nanoparticles for drug delivery and biosensing applications. Adv. Funct. Mater. 2007, 17, 1225-1236.

[20] Fang, X. L.; Zhao, X. J.; Fang, W. J.; Chen, C.; Zheng, N. F. Selftemplating synthesis of hollow mesoporous silica and their applications in catalysis and drug delivery. Nanoscale 2013, 5, 2205-2218.

[21] Xu, Z. G.; Ma, X. Q.; Gao, Y. E.; Hou, M. L.; Xue, P.; Li, C. M.; Kang, Y. J. Multifunctional silica nanoparticles as a promising theranostic platform for biomedical applications. Mater. Chem. Front. 2017, 1, 1257-1272.

[22] Zhu, Y. F.; Shi, J. L.; Li, Y. S.; Chen, H. R.; Shen, W. H.; Dong, X. P. Storage and release of ibuprofen drug molecules in hollow mesoporous silica spheres with modified pore surface. Microporous Mesoporous Mater. 2005, 85, 75-81.

[23] Vallet-Regi, M.; Rámila, A.; del Real, R. P.; Pérez-Pariente, J. A new property of MCM-41: Drug delivery system. Chem. Mater. 2011, 13, 308-311.

[24] Zhu, Y. F.; Shi, J. L.; Shen, W. H.; Chen, H. R.; Dong, X. P.; Ruan, M. L. Preparation of novel hollow mesoporous silica spheres and their sustained-release property. Nanotechnology 2005, 16, 2633-2638.

[25] Li, Y. H.; Li, N.; Pan, W.; Yu, Z. Z.; Yang, L. M.; Tang, B. Hollow mesoporous silica nanoparticles with tunable structures for controlled drug delivery. ACS Appl. Mater. Interfaces 2017, 9, 2123-2129.

[26] Wibowo, D.; Zhao, C. X.; Peters, B. C.; Middelberg, A. P. J. Sustained release of fipronil insecticide in vitro and in vivo from biocompatible silica nanocapsules. J. Agric. Food Chem. 2014, 62, 12504-12511.

[27] Gao, Y.; Chen, Y.; Ji, X. F.; He, X. Y.; Yin, Q.; Zhang, Z. W.; Shi, J. L.; Li, Y. P. Controlled intracellular release of doxorubicin in multidrug-resistant cancer cells by tuning the shell-pore sizes of mesoporous silica nanoparticles. ACS Nano 2011, 5, 9788-9798.

[28] Jiao, Y. F.; Guo, J.; Shen, S.; Chang, B. S.; Zhang, Y. H.; Jiang, X. G.; Yang, W. L. Synthesis of discrete and dispersible hollow mesoporous silica nanoparticles with tailored shell thickness for controlled drug release. J. Mater. Chem. 2012, 22, 17636-17643.

[29] Jung, Y.; Huh, Y.; Kim, D. Recent advances in surface engineering of porous silicon nanomaterials for biomedical applications. Microporous Mesoporous Mater. 2021, 310, 110673

[30] Li, W.; Liu, Z. H.; Fontana, F.; Ding, Y. P.; Liu, D. F.; Hirvonen, J. T.; Santos, H. A. Tailoring porous silicon for biomedical applications: From drug delivery to cancer immunotherapy. Adv. Mater. 2018, 30, 1703740 .
[31] Yang, Y. N.; Zhang, M.; Song, H.; Yu, C. Z. Silica-Based nanoparticles for biomedical applications: From nanocarriers to biomodulators. Acc. Chem. Res. 2020, 53, 1545-1556.

[32] Ahmad Nor, Y.; Niu, Y. T.; Karmakar, S.; Zhou, L.; Xu, C.; Zhang, J.; Zhang, H. W.; Yu, M. H.; Mahony, D.; Mitter, N. et al. Shaping nanoparticles with hydrophilic compositions and hydrophobic properties as nanocarriers for antibiotic delivery. ACS Cent. Sci. 2015, 1, 328-334.

[33] Niu, Y. T.; Yu, M. H.; Hartono, S. B.; Yang, J.; Xu, H. Y.; Zhang, H. W.; Zhang, J.; Zou, J.; Dexter, A.; Gu, W. Y. et al. Nanoparticles mimicking viral surface topography for enhanced cellular delivery. Adv. Mater. 2013, 25, 6233-6237.

[34] Song, H.; Ahmad Nor, Y.; Yu, M. H.; Yang, Y. N.; Zhang, J.; Zhang, H. W.; Xu, C.; Mitter, N.; Yu, C. Z. Silica nanopollens enhance adhesion for long-term bacterial inhibition. J. Am. Chem. Soc. 2016, 138, 6455-6462.

[35] Zhu, Y. F.; Shi, J. L.; Shen, W. H.; Dong, X. P.; Feng, J. W.; Ruan, M. L.; Li, Y. S. Stimuli-responsive controlled drug release from a hollow mesoporous silica sphere/polyelectrolyte multilayer core-shell structure. Angew. Chem., Int. Ed. 2005, 44, 5083-5087.

[36] Hao, N. J.; Jayawardana, K. W.; Chen, X.; Yan, M. D. One-step synthesis of amine-functionalized hollow mesoporous silica nanoparticles as efficient antibacterial and anticancer materials. ACS Appl. Mater. Interfaces 2015, 7, 1040-1045.

[37] Cheng, K. W.; Zhang, Y.; Li, Y. J.; Gao, Z. G.; Chen, F. H.; Sun, K.; An, P. J.; Sun, C.; Jiang, Y.; Sun, B. W. A novel pH-responsive hollow mesoporous silica nanoparticle (HMSN) system encapsulating doxorubicin (DOX) and glucose oxidase (GOX) for potential cancer treatment. J. Mater. Chem. B 2019, 7, 3291-3302.

[38] Chen, Y.; Chen, H. R.; Zeng, D. P.; Tian, Y. B.; Chen, F.; Feng, J. W.; Shi, J. L. Core/shell structured hollow mesoporous nanocapsules: A potential platform for simultaneous cell Imaging and anticancer drug delivery. ACS Nano 2010, 4, 6001-6013.

[39] Zhao, W. R.; Chen, H. R.; Li, Y. S.; Li, L.; Lang, M. D.; Shi, J. L. Uniform rattle-type hollow magnetic mesoporous spheres as drug delivery carriers and their sustained-release property. Adv. Funct. Mater. 2008, 18, 2780-2788.

[40] Jia, X. M.; Yang, Z. Y.; Wang, Y. J.; Chen, Y.; Yuan, H. T.; Chen, H. Y.; Xu, X. X.; Gao, X. Q.; Liang, Z. Z.; Sun, Y. et al. Hollow mesoporous silica@metal-organic framework and applications for pH-responsive drug delivery. ChemMedChem 2018, 13, 400-405.

[41] Wu, M. Y.; Chen, Y.; Zhang, L. X.; Li, X. Y.; Cai, X. J.; Du, Y. Y.; Zhang, L. L.; Shi, J. L. A salt-assisted acid etching strategy for hollow mesoporous silica/organosilica for $\mathrm{pH}$-responsive drug and gene co-delivery. J. Mater. Chem. B 2015, 3, 766-775.

[42] Teng, Z. G.; Wang, C. Y.; Tang, Y. X.; Li, W.; Bao, L.; Zhang, X. H.; Su, X. D.; Zhang, F.; Zhang, J. J.;Wang, S. J. et al. Deformable Hollow periodic mesoporous organosilica nanocapsules for significantly improved cellular uptake. J. Am. Chem. Soc. 2018, 140, 1385-1393.

[43] Zhang, J. J.; Lu, N.; Weng, L. X.; Feng, Z. H.; Tao, J.; Su, X. D.; Yu, R. F.; Shi, W. H.; Qiu, Q.; Teng, Z. G. et al. General and facile syntheses of hybridized deformable hollow mesoporous organosilica nanocapsules for drug delivery. J. Colloid Interface Sci. 2021, 583, 714-721.

[44] Fan, W. P.; Lu, N.; Shen, Z. Y.; Tang, W.; Shen, B.; Cui, Z. W.; Shan, L. L.; Yang, Z.; Wang, Z. T.; Jacobson, O. et al. Generic synthesis of small-sized hollow mesoporous organosilica nanoparticles for oxygenindependent X-ray-activated synergistic therapy. Nat. Coтmun. 2019, $10,1241$.

[45] Teng, Z. G.; Li, W.; Tang, Y. X.; Elzatahry, A.; Lu, G. M.; Zhao, D. Y. Mesoporous organosilica hollow nanoparticles: Synthesis and applications. Adv. Mater. 2019, 31, 1707612.

[46] Chen, Y.; Meng, Q. S.; Wu, M. Y.; Wang, S. G.; Xu, P. F.; Chen, H. R.; Li, Y. P.; Zhang, L. X.; Wang, L. Z.; Shi, J. L. Hollow mesoporous organosilica nanoparticles: A generic intelligent framework-hybridization approach for biomedicine. J. Am. Chem. Soc. 2014, 136, 1632616334.

[47] Yang, Y. N.; Lu, Y.; Abbaraju, P. L.; Zhang, J.; Zhang, M.; Xiang, G. Y.; Yu, C. Z. Multi-shelled dendritic mesoporous organosilica hollow spheres: Roles of composition and architecture in cancer immunotherapy. Angew. Chem., Int. Ed. 2017, 56, 8446-8450.

[48] Yu, X. F.; Li, W. C.; Hu, Y. R.; Ye, C. Y.; Lu, A. H. Sculpturing solid 
polymer spheres into internal gridded hollow carbon spheres under controlled pyrolysis micro-environment. Nano Res. 2021, 14, 1565-1573.

[49] Ahmad Nor, Y.; Zhang, H. W.; Purwajanti, S.; Song, H.; Meka, A. K.; Wang, Y.; Mitter, N.; Mahony, D.; Yu, C. Z. Hollow mesoporous carbon nanocarriers for vancomycin delivery: Understanding the structure-release relationship for prolonged antibacterial performance. J. Mater. Chem. B 2016, 4, 7014-7021.

[50] Sercombe, L.; Veerati, T.; Moheimani, F.; Wu, S. Y.; Sood, A. K.; Hua, S. Advances and challenges of liposome assisted drug delivery. Front. Pharmacol. 2015, 6, 286.

[51] Vlasova, K. Y.; Piroyan, A.; Le-Deygen, I. M.; Vishwasrao, H. M.; Ramsey, J. D.; Klyachko, N. L.; Golovin, Y. I.; Rudakovskaya, P. G.; Kireev, I. I.; Kabanov, A. V. et al. Magnetic liposome design for drug release systems responsive to super-low frequency alternating current magnetic field (AC MF). J. Colloid. Interface Sci. 2019, 552, 689-700.

[52] Kirjavainen, M.; Urtti, A.; Valjakka-Koskela, R.; Kiesvaara, J.; Mönkkönen, J. Liposome-skin interactions and their effects on the skin permeation of drugs. Eur. J. Pharm. Sci. 1999, 7, 279-286.

[53] He, H. S.; Lu, Y.; Qi, J. P.; Zhu, Q. G.; Chen, Z. J.; Wu, W. Adapting liposomes for oral drug delivery. Acta Pharm. Sin. B 2019, 9, 36-48.

[54] Shi, D.; Mi, G. J.; Shen, Y.; Webster, T. J. Glioma-targeted dual functionalized thermosensitive ferri-liposomes for drug delivery through an in vitro blood-brain barrier. Nanoscale 2019, 11, 1505715071.

[55] Kamaly, N.; Yameen, B.; Wu, J.; Farokhzad, O. C. Degradable controlled-release polymers and polymeric nanoparticles: Mechanisms of controlling drug release. Chem. Rev. 2016, 116, 2602-2663.

[56] Shim, Y. B.; Jung, H. H.; Jang, J. W.; Yang, H. S.; Bae, H.; Park, J. C.; Choi, B.; Lee, S. H. Fabrication of hollow porous PLGA microspheres using sucrose for controlled dual delivery of dexamethasone and BMP2. J. Ind. Eng. Chem. 2016, 37, 101-106.

[57] Ke, C. J.; Su, T. Y.; Chen, H. L.; Liu, H. L.; Chiang, W. L.; Chu, P. C.; Xia, Y. N.; Sung, H. W. Smart multifunctional hollow microspheres for the quick release of drugs in intracellular lysosomal compartments. Angew. Chem., Int. Ed. 2011, 50, 8086-8089.

[58] Liu, Y. S.; Wu, H. L.; Jia, Z.; Du, B.; Liu, D. Y.; Zhou, Z. M. Silk fibroin-modified ploylactic acid-glycolic acid copolymer porous microspheres as gingival mesenchymal stem cells delivery carrier. Chem. Res. Chin. Univ. 2019, 40, 2419-2426.

[59] Zhang, X. Y.; Han, L.; Liu, M. Y.; Wang, K.; Tao, L.; Wan, Q.; Wei, Y. Recent progress and advances in redox-responsive polymers as controlled delivery nanoplatforms. Mater. Chem. Front. 2017, 1, 807-822.

[60] Wu, J. E.; Zhang, Z. X.; Gu, J. G.; Zhou, W. X.; Liang, X. Y.; Zhou, G. Q.; Han, C. C.; Xu, S. S.; Liu, Y. Mechanism of a long-term controlled drug release system based on simple blended electrospun fibers. J. Controlled Release 2020, 320, 337-346.

[61] Li, G. L.; Yang, X. Y.; Wang, B.; Wang, J. Y.; Yang, X. L. Monodisperse temperature-responsive hollow polymer microspheres: Synthesis, characterization and biological application. Polymer 2008, 49, 3436-3443.

[62] Yang, X. Y.; Chen, L. T.; Huang, B.; Bai, F.; Yang, X. L. Synthesis of $\mathrm{pH}$-sensitive hollow polymer microspheres and their application as drug carriers. Polymer 2009, 50, 3556-3563.

[63] Shi, J. J.; Xiao, Z. Y.; Votruba, A. R.; Vilos, C.; Farokhzad, O. C. Differentially charged hollow core/shell lipid-polymer-lipid hybrid nanoparticles for small interfering rna delivery. Angew. Chem., Int. Ed. 2011, 50, 7027-7031.

[64] Wu, Y. N.; Zhou, M. M.; Li, S.; Li, Z. H.; Li, J.; Wu, B. Z.; Li, G. T.; Li, F. T.; Guan, X. H. Magnetic metal-organic frameworks: $\gamma$ $\mathrm{Fe}_{2} \mathrm{O}_{3} @$ MOFs via confined in situ pyrolysis method for drug delivery. Small 2014, 10, 2927-2936.

[65] Bag, P. P.; Wang, D.; Chen, Z.; Cao, R. Outstanding drug loading capacity by water stable microporous MOF: A potential drug carrier. Chem. Commun. 2016, 52, 3669-3672.

[66] Gao, X. C.; Cui, R. X.; Song, L. J.; Liu, Z. L. Hollow structural metal-organic frameworks exhibit high drug loading capacity, targeted delivery and magnetic resonance/optical multimodal imaging. Dalton Trans. 2019, 48, 17291-17297.

[67] Sun, X.; He, G. H.; Xiong, C. X.; Wang, C. Y.; Lian, X.; Hu, L. F.;
Li, Z. K.; Dalgarno, S. J.; Yang, Y. W.; Tian, J. One-pot fabrication of hollow porphyrinic MOF nanoparticles with ultrahigh drug loading toward controlled delivery and synergistic cancer therapy. ACS Appl. Mater. Interfaces 2021, 13, 3679-3693.

[68] Cui, R. X.; Zhao, P. F.; Yan, Y. L.; Bao, G.; Damirin, A.; Liu, Z. L. Outstanding drug-loading/release capacity of hollow Fe-metalorganic framework-based microcapsules: A potential multifunctional drug-delivery platform. Inorg. Chem. 2021, 60, 1664-1671.

[69] Mao, D.; Wan, J. W.; Wang, J. Y.; Wang, D. Sequential templating approach: A groundbreaking strategy to create hollow multishelled structures. Adv. Mater. 2019, 31, 1802874.

[70] Wang, J. Y.; Wan, J. W.; Wang, D. Hollow multishelled structures for promising applications: Understanding the structure-performance correlation. Acc. Chem. Res. 2019, 52, 2169-2178.

[71] Wang, J. Y.; Wan, J. W.; Yang, N. L.; Li, Q.; Wang, D. Hollow multishell structures exercise temporal-spatial ordering and dynamic smart behaviour. Nat. Rev. Chem. 2020, 4, 159-168.

[72] Ma, X. M.; Zhang, X. T.; Yang, L.; Wang, G.; Jiang, K.; Wu, G.; Cui, W. G.; Wei, Z. P. Tunable construction of multi-shelled hollow carbonate nanospheres and their potential applications. Nanoscale 2016, 8 , $8687-8695$.

[73] Wu, L.; Zhang, H. J.; Wu, M. H.; Zhong, Y. F.; Liu, X. W.; Jiao, Z. Dual-templating synthesis of multi-shelled mesoporous silica nanoparticles as catalyst and drug carrier. Microporous Mesoporous Mater. 2016, 228, 318-328.

[74] Huang, C. C.; Huang, W.; Yeh, C. S. Shell-by-shell synthesis of multi-shelled mesoporous silica nanospheres for optical imaging and drug delivery. Biomaterials 2011, 32, 556-564.

[75] Teng, Z. G.; Su, X. D.; Zheng, Y. Y.; Zhang, J. J.; Liu, Y.; Wang, S. J.; Wu, J.; Chen, G. T.; Wang, J. D.; Zhao, D. Y. et al. A facile multi-interface transformation approach to monodisperse multipleshelled periodic mesoporous organosilica hollow spheres. $\mathrm{J}$. Am. Chem. Soc. 2015, 137, 7935-7944.

[76] Zhao, D. C.; Yang, N. L.; Wei, Y.; Jin, Q.; Wang, Y. L.; He, H. Y.; Yang, Y.; Han, B.; Zhang, S. J.; Wang, D. Sequential drug release via chemical diffusion and physical barriers enabled by hollow multishelled structures. Nat. Commun. 2020, 11, 4450.

[77] Dehghani, E.; Salami-Kalajahi, M.; Roghani-Mamaqani, H. Fabricating cauliflower-like and dumbbell-like Janus particles: Loading and simultaneous release of DOX and ibuprofen. Colloids Surf. B Biointerfaces 2019, 173, 155-163.

[78] Li, X. M.; Zhou, L.; Wei, Y.; Mohamed El-Toni, A.; Zhang, F.; Zhao, D. Y. Anisotropic growth-induced synthesis of dual-compartment Janus mesoporous silica nanoparticles for bimodal triggered drugs delivery. J. Am. Chem. Soc. 2014, 136, 15086-15092.

[79] Li, X. M.; Zhou, L.; Wei, Y.; El-Toni, A. M.; Zhang, F.; Zhao, D. Y. Anisotropic encapsulation-induced synthesis of asymmetric single-hole mesoporous nanocages. J. Am. Chem. Soc. 2015, 137, 5903-5906.

[80] Yoshida, M.; Roh, K. H.; Mandal, S.; Bhaskar, S.; Lim, D.; Nandivada, H.; Deng, X. P.; Lahann, J. Structurally controlled biohybrid materials based on unidirectional association of anisotropic microparticles with human endothelial cells. Adv. Mater. 2009, 21 , 4920-4925.

[81] Wu, L. Y.; Ross, B. M.; Hong, S. G.; Lee, L. P. Bioinspired nanocorals with decoupled cellular targeting and sensing functionality. Small 2010, 6, 503-507.

[82] Chen, X. J.; Zhang, X. P.; Li, S. N.; Zhang, L. Y.; Zhang, Q.; Chen, Z. H.; Li, L.; Su, Z. M.; Cheng, S. Q.; Wang, C. G. Engineering of Yin Yang-like nanocarriers for varisized guest delivery and synergistic eradication of patient-derived hepatocellular carcinoma. Nanoscale Horiz. 2019, 4, 1046-1055.

[83] Zhang, S. L.; Chu, Z. Q.; Yin, C.; Zhang, C. Y.; Lin, G.; Li, Q. Controllable drug release and simultaneously carrier decomposition of $\mathrm{SiO}_{2}$-drug composite nanoparticles. J. Am. Chem. Soc. 2013, 135, 5709-5716.

[84] Doan, T. V. P.; Couet, W.; Olivier, J. C. Formulation and in vitro characterization of inhalable rifampicin-loaded PLGA microspheres for sustained lung delivery. Int. J. Pharm. 2011, 414, 112-117.

[85] Wang, Z. J.; Qian, L.; Wang, X. L.; Yang, F.; Yang, X. R. Construction of hollow DNA/PLL microcapsule as a dual carrier for controlled delivery of DNA and drug. Colloids Sur. A Physicochem. Eng. Asp. 2008, 326, 29-36. 
[86] Zhao, Y.; Lin, L. N.; Lu, Y.; Chen, S. F.; Dong, L.; Yu, S. H. Templating synthesis of preloaded doxorubicin in hollow mesoporous silica nanospheres for biomedical applications. Adv. Mater. 2010, $22,5255-5259$

[87] Limnell, T.; Santos, H. A.; Mäkilä, E.; Heikkilä, T.; Salonen, J.; Murzin, D. Y.; Kumar, N.; Laaksonen, T.; Peltonen, L.; Hirvonen, J. Drug delivery formulations of ordered and nonordered mesoporous silica: Comparison of three drug loading methods. J. Pharm. Sci. 2011, 100, 3294-3306.

[88] Rayamajhi, S.; Marchitto, J.; Nguyen, T. D. T.; Marasini, R.; Celia, C.; Aryal, S. pH-responsive cationic liposome for endosomal escape mediated drug delivery. Colloids Surf. B Biointerfaces 2020, 188 , 110804

[89] Xu, Y. H.; Szoka, F. C. Mechanism of DNA release from cationic liposome/DNA complexes used in cell transfection. Biochemistry 1996, 35, 5616-5623.

[90] Chen, P.; Wang, Z. Y.; Zong, S. F.; Zhu, D.; Chen, H.; Zhang, Y. Z.; $\mathrm{Wu}, \mathrm{L}$.; Cui, Y. P. pH-sensitive nanocarrier based on gold/silver core-shell nanoparticles decorated multi-walled carbon manotubes for tracing drug release in living cells. Biosens. Bioelectron. 2016, 75, 446-451.

[91] Huo, T. T.; Yang, Y. F.; Qian, M.; Jiang, H. L.; Du, Y. L.; Zhang, X. Y.; Xie, Y. B.; Huang, R. Q. Versatile hollow cof nanospheres via manipulating transferrin corona for precise glioma-targeted drug delivery. Biomaterials 2020, 260, 120305.

[92] Springer, U. S. Chemical Bond. In Encyclopedia of Microfluidics and Nanofluidics. Li, D. Q., Ed.; Springer: Boston, 2008.

[93] Su, Z. H.; Liang, Y. C.; Yao, Y.; Wang, T. Q.; Zhang, N. Polymeric complex micelles based on the double-hydrazone linkage and dual drug-loading strategy for $\mathrm{pH}$-sensitive docetaxel delivery. J. Mater. Chem. B 2016, 4, 1122-1133.

[94] Quang Tran, H.; Bhave, M.; Yu, A. M. Current advances of hollow capsules as controlled drug delivery systems. ChemistrySelect 2020, $5,5537-5551$.

[95] Ngamcherdtrakul, W.; Morry, J.; Gu, S. D.; Castro, D. J.; Goodyear, S. M.; Sangvanich, T.; Reda, M. M.; Lee, R.; Mihelic, S. A.; Beckman, B. L. et al. Cationic polymer modified mesoporous silica nanoparticles for targeted sirna delivery to HER $2^{+}$breast cancer. Adv. Funct. Mater. 2015, 25, 2646-2659.

[96] Patel, J. K.; Patel, A. P. Passive Targeting of nanoparticles to cancer. In Surface Modification of Nanoparticles for Targeted Drug Delivery. Pathak, Y. V., Ed.; Cham: Springer, 2019; pp 125-143.

[97] Gocheva, G.; Ivanova, A. A look at receptor-ligand pairs for activetargeting drug delivery from crystallographic and molecular dynamics perspectives. Mol. Pharmaceutics 2019, 16, 3293-3321.

[98] Park, J. Y.; Park, S.; Lee, T. S.; Hwang, Y. H.; Kim, J. Y.; Kang, W. J.; Key, J. Biodegradable micro-sized discoidal polymeric particles for lung-targeted delivery system. Biomaterials 2019, 218, 119331.

[99] Baboci, L.; Capolla, S.; Di Cintio, F.; Colombo, F.; Mauro, P.; Dal Bo, M.; Argenziano, M.; Cavalli, R.; Toffoli, G.; Macor, P. The dual role of the liver in nanomedicine as an actor in the elimination of nanostructures or a therapeutic target. J. Oncol. 2020, 2020, 4638192.

[100] Zhang, X. Y.; Wang, H.; Fang, Y.; Fan, Y. Stimuli-responsive $\mathrm{Fe}_{3} \mathrm{O}_{4}$ nanoparticle modified by conjugated linoleic acid. Chem. J. Chin. Univ. 2020, 41, 2519-2525.

[101] Yang, C.; Song, G. S.; Yuan, H. F.; Yang, Y.; Wang, Y. Q.; Ye, D. J.; Meng, H. M.; Huan, S. Y.; Zhang, X. B. Manganese-fluorouracil metallodrug nanotheranostic for MRI-correlated drug release and enhanced chemoradiotherapy. CCS Chem. 2020, 3, 1116-1128.

[102] Liu, D. H.; Du, J. S.; Qi, S. L.; Li, M. Y.; Wang, J. F.; Liu, M. H.; Du, X. L.; Wang, X. Y.; Ren, B. C.; Wu, D. et al. Supramolecular nanoparticles constructed from pillar[5]arene-based host-guest complexation with enhanced aggregation-induced emission for imaging-guided drug delivery. Mater. Chem. Front. 2021, 5, $1418-1427$.

[103] Yang, B.; Zhang, X. D.; Li, J.; Tian, J.; Wu, Y. P.; Yu, F. X.; Wang, R. B.; Wang, H.; Zhang, D. W.; Liu, Y. et al. In situ loading and delivery of short single- and double-stranded DNA by supramolecular organic frameworks. CCS Chem. 2019, 1, 156-165.

[104] Wu, M. X.; Yan, H. J.; Gao, J.; Cheng, Y.; Yang, J.; Wu, J. R.; Gong, B. J.; Zhang, H. Y.; Yang, Y. W. Multifunctional supramolecular materials constructed from polypyrrole@UiO-66 nanohybrids and pillararene nanovalves for targeted chemophotothermal therapy. ACS Appl. Mater. Interfaces 2018, 10, 34655-34663.

[105] Gao, J.; Li, J.; Geng, W. C.; Chen, F. Y.; Duan, X. C.; Zheng, Z.; Ding, D.; Guo, D. S. Biomarker displacement activation: A general host-guest strategy for targeted phototheranostics in vivo. $\mathrm{J}$. Am. Chem. Soc. 2018, 140, 4945-4953.

[106] Ren, Y. S.; Guo, Y. Y.; Liu, X. Y.; Song, J.; Zhang, C. Platinum(IV) prodrug-grafted phosphorothioate DNA and its self-assembled nanostructure for targeted drug delivery. Chem. J. Chin. Univ. 2020, $41,1721-1730$.

[107] Liang, Q.; Xi, J. Q.; Gao, X. J.; Zhang, R. F.; Yang, Y. L.; Gao, X. F.; Yan, X. Y.; Gao, L. Z.; Fan, K. L. A metal-free nanozyme-activated prodrug strategy for targeted tumor catalytic therapy. Nano Today 2020, 35, 100935.

[108] An, J.; Hu, Y. G.; Cheng, K.; Li, C.; Hou, X. L.; Wang, G. L.; Zhang, X. S.; Liu, B.; Zhao, Y. D.; Zhang, M. Z. ROS-augmented and tumor-microenvironment responsive biodegradable nanoplatform for enhancing chemo-sonodynamic therapy. Biomaterials 2020, 234, 119761.

[109] Domiński, A.; Konieczny, T.; Duale, K.; Krawczyk, M.; PastuchGawolek, G.; Kurcok, P. Stimuli-responsive aliphatic polycarbonate nanocarriers for tumor-targeted drug delivery. Polymers 2020, 12, 2890.

[110] Hao, N. J.; Chen, X.; Jeon, S.; Yan, M. D. Carbohydrate- conjugated hollow oblate mesoporous silica nanoparticles as nanoantibiotics to target mycobacteria. Adv. Healthc. Mater. 2015, 4, 2797-2801.

[111] Yan, S. Z.; Chen, S.; Gou, X. B.; Yang, J.; An, J. X.; Jin, X. Y.; Yang, Y. W.; Chen, L.; Gao, H. Biodegradable supramolecular materials based on cationic polyaspartamides and pillar[5]arene for targeting gram-positive bacteria and mitigating antimicrobial resistance. Adv. Funct. Mater. 2019, 29, 1904683.

[112] Yue, Z. G.; Wei, W.; You, Z. X.; Yang, Q. Z.; Yue, H.; Su, Z. G.; $\mathrm{Ma}, \mathrm{G}$. H. Iron oxide nanotubes for magnetically guided delivery and $\mathrm{pH}$-activated release of insoluble anticancer drugs. Adv. Funct. Mater. 2011, 21, 3446-3453.

[113] Li, X. S.; Lovell, J. F.; Yoon, J.; Chen, X. Y. Clinical development and potential of photothermal and photodynamic therapies for cancer. Nat. Rev. Clin. Oncol. 2020, 17, 657-674.

[114] Gao, F.; Tang, Y.; Liu, W. L.; Zou, M. Z.; Huang, C.; Liu, C. J.; Zhang, X. Z. Intra/extracellular lactic acid exhaustion for synergistic metabolic therapy and immunotherapy of tumors. Adv. Mater. 2019, 31,1904639

[115] Manzari, M. T.; Shamay, Y.; Kiguchi, H.; Rosen, N.; Scaltriti, M.; Heller, D. A. Targeted drug delivery strategies for precision medicines. Nat. Rev. Mater. 2021, 6, 351-370.

[116] Jiang, T. Y.; Mo, R.; Bellotti, A.; Zhou, J. P.; Gu, Z. Gel-liposomemediated co-delivery of anticancer membrane-associated proteins and small-molecule drugs for enhanced therapeutic efficacy. $A d v$. Funct. Mater. 2014, 24, 2295-2304.

[117] Wang, Y. Y.; Wang, W. L.; Shen, X. C.; Zhou, B.; Chen, T.; Guo, Z. X.; Wen, C. C.; Jiang, B. P.; Liang, H. Combination-responsive $\mathrm{MoO}_{3-x}$-hybridized hyaluronic acid hollow nanospheres for cancer phototheranostics. ACS Appl. Mater. Interfaces 2018, 10, 4208842101.

[118] Yang, G. B.; Xu, L. G.; Xu, J.; Zhang, R.; Song, G. S.; Chao, Y.; Feng, L. Z.; Han, F. X.; Dong, Z. L.; Li, B. et al. Smart nanoreactors for $\mathrm{pH}$-responsive tumor homing, mitochondria-targeting, and enhanced photodynamic-immunotherapy of cancer. Nano Lett. 2018, 18, 2475-2484.

[119] Jin, L.; Huang, Q. J.; Zeng, H. Y.; Du, J. Z.; Xu, S.; Chen, C. R. Hydrotalcite-gated hollow mesoporous silica delivery system for controlled drug release. Microporous Mesoporous Mater. 2019, 274, 304-312.

[120] Liu, J.; Chen, B.; Zhang, J. J. Preparation of pH-responsive doxorubicin nanocapsules by combining high-gravity antisolvent precipitation with in-situ polymerization for intracellular anticancer drug delivery. Chem. Res. Chin. Univ. 2020, 36, 927-933.

[121] Dong, Z. L.; Feng, L. Z.; Hao, Y.; Chen, M. C.; Gao, M.; Chao, Y.; Zhao, H.; Zhu, W. W.; Liu, J. J.; Liang, C. et al. Synthesis of hollow biomineralized $\mathrm{CaCO}_{3}$-polydopamine nanoparticles for multimodal imaging-guided cancer photodynamic therapy with reduced skin photosensitivity. J. Am. Chem. Soc. 2018, 140, 2165-2178. 
[122] Yang, J. H.; Gao, F.; Han, D. L.; Yang, L. L.; Kong, X. W.; Wei, M. B.; Cao, J.; Liu, H. L.; Wu, Z. T.; Pan, G. Y. Multifunctional zinc-based hollow nanoplatforms as a smart ph-responsive drug delivery system to enhance in vivo tumor-inhibition efficacy. Mater. Des. 2018, 139, 172-180.

[123] Yang, G. B.; Xu, L. G.; Chao, Y.; Xu, J.; Sun, X. Q.; Wu, Y. F.; Peng, R.; Liu, Z. Hollow $\mathrm{MnO}_{2}$ as a tumor-microenvironmentresponsive biodegradable nano-platform for combination therapy favoring antitumor immune responses. Nat. Commun. 2017, 8 , 902.

[124] Weerasuriya, D. R. K.; Wijesinghe, W. P. S. L.; Rajapakse, R. M. G. Encapsulation of anticancer drug copper bis(8-hydroxyquinoline) in hydroxyapatite for $\mathrm{pH}$-sensitive targeted delivery and slow release. Mater. Sci. Eng. C 2017, 71, 206-213.

[125] Kocak, G.; Tuncer, C.; Bütün, V. pH-responsive polymers. Polym. Chem. 2017, 8, 144-176.

[126] Zhang, L. Y.; Zhang, M. J.; Zhou, L.; Han, Q. H.; Chen, X. J.; Li, S. N.; Li, L.; Su, Z. M.; Wang, C. G. Dual drug delivery and sequential release by amphiphilic Janus nanoparticles for liver cancer theranostics. Biomaterials 2018, 181, 113-125.

[127] Gao, M. M.; Wang, C. L.; Dou, H. J.; Xu, G. X. One-step selfassembly/polymerization fabrication and biomedical application of carboplatin@dextran nanocarrier. Chem.J. Chin. Univ. 2019, 40, 1304-1309.

[128] Lu, L. L.; Xiong, W. Y.; Ma, J. B.; Gao, T. F.; Peng, S. Y.; Xiao, W. Design of dual-responsive nanocarries with high drug loading capacity based on hollow mesoporous organosilica nanoparticles. Mater. Chem. Phys. 2019, 223, 230-235.

[129] Zhao, N. N.; Lin, X. Y.; Zhang, Q.; Ji, Z. X.; Xu, F. J. Redoxtriggered gatekeeper-enveloped starlike hollow silica nanoparticles for intelligent delivery systems. Small 2015, 11, 6467-6479.

[130] Zhao, Q. F.; Wang, S. Y.; Yang, Y.; Li, X.; Di, D. H.; Zhang, C. G.; Jiang, T. Y.; Wang, S. L. Hyaluronic acid and carbon dots-gated hollow mesoporous silica for redox and enzyme-triggered targeted drug delivery and bioimaging. Mater. Sci. Eng. C 2017, 78, 475-484.

[131] Wu, M. Y.; Meng, Q. S.; Chen, Y.; Zhang, L. X.; Li, M. L.; Cai, X. J.; Li, Y. P.; Yu, P. C.; Zhang, L. L.; Shi, J. L. Large pore-sized hollow mesoporous organosilica for redox-responsive gene delivery and synergistic cancer chemotherapy. Adv. Mater. 2016, 28, 19631969.

[132] Jiao, J.; Li, X.; Zhang, S.; Liu, J.; Di, D. H.; Zhang, Y.; Zhao, Q. F.; Wang, S. L. Redox and $\mathrm{pH}$ dual-responsive peg and chitosanconjugated hollow mesoporous silica for controlled drug release. Mater. Sci. Eng. C 2016, 67, 26-33.

[133] Tian, Y.; Guo, R. R.; Jiao, Y. F.; Sun, Y. F.; Shen, S.; Wang, Y. J.; Lu, D. R.; Jiang, X. G.; Yang, W. L. Redox stimuli-responsive hollow mesoporous silica nanocarriers for targeted drug delivery in cancer therapy. Nanoscale Horiz. 2016, 1, 480-487.

[134] Hadipour Moghaddam, S. P.; Yazdimamaghani, M.; Ghandehari, H. Glutathione-sensitive hollow mesoporous silica nanoparticles for controlled drug delivery. J. Controlled Release 2018, 282, 62-75.

[135] Zhang, Y. Y.; Ang, C. Y.; Li, M. H.; Tan, S. Y.; Qu, Q. Y.; Luo, Z.; Zhao, Y. L. Polymer-coated hollow mesoporous silica nanoparticles for triple-responsive drug delivery. ACS Appl. Mater. Interfaces 2015, 7, 18179-18187.

[136] Zhu, Y. F.; Meng, W. J.; Gao, H.; Hanagata, N. Hollow mesoporous silica/poly(L-lysine) particles for codelivery of drug and gene with enzyme-triggered release property. J. Phys. Chem. C 2011, $115,13630-13636$.

[137] Kaziem, A. E.; Gao, Y. H.; He, S.; Li, J. H. Synthesis and insecticidal activity of enzyme-triggered functionalized hollow mesoporous silica for controlled release. J. Agric. Food Chem. 2017, 65, 7854-7864.

[138] Liu, C. Q.; Chen, Z. W.; Wang, Z. Z.; Li, W.; Ju, E. G.; Yan, Z. Q.; Liu, Z.; Ren, J. S.; Qu, X. G. A graphitic hollow carbon nitride nanosphere as a novel photochemical internalization agent for targeted and stimuli-responsive cancer therapy. Nanoscale 2016, 8 , 12570-12578.

[139] Zhang, J. F.; Zhang, J.; Li, W. Y.; Chen, R.; Zhang, Z. Y.; Zhang, W. J.; Tang, Y. B.; Chen, X. Y.; Liu, G.; Lee, C. S. Degradable hollow mesoporous silicon/carbon nanoparticles for photoacoustic imaging-guided highly effective chemo-thermal tumor therapy in vitro and in vivo. Theranostics 2017, 7, 3007-3020.

[140] Wu, F.; Zhang, M.; Lu, H. W.; Liang, D.; Huang, Y. L.; Xia, Y. H.; Hu, Y. Q.; Hu, S. Q.; Wang, J. X.; Yi, X. Y. et al. Triple stimuliresponsive magnetic hollow porous carbon-based nanodrug delivery system for magnetic resonance imaging-guided synergistic photothermal/chemotherapy of cancer. ACS Appl. Mater. Interfaces 2018, 10, 21939-21949.

[141] Deng, X. R.; Li, K.; Cai, X. C.; Liu, B.; Wei, Y.; Deng, K. R.; Xie, Z. X.; Wu, Z. J.; Ma, P. A.; Hou, Z. Y. et al. A hollow-structured $\mathrm{CuS} @ \mathrm{Cu}_{2} \mathrm{~S} @ A$ Au nanohybrid: Synergistically enhanced photothermal efficiency and photoswitchable targeting effect for cancer theranostics. Adv. Mater. 2017, 29, 1701266.

[142] Zhou, L.; Chen, Z. W.; Dong, K.; Yin, M. L.; Ren, J. S.; Qu, X. G. DNA-mediated construction of hollow upconversion nanoparticles for protein harvesting and near-infrared light triggered release. Adv. Mater. 2014, 26, 2424-2430.

[143] Cho, H. J.; Chung, M.; Shim, M. S. Engineered photo-responsive materials for near-infrared-triggered drug delivery. J. Ind. Eng. Chem. 2015, 31, 15-25.

[144] Zhao, L. Z.; Peng, J. J.; Huang, Q.; Li, C. Y.; Chen, M.; Sun, Y.; Lin, Q. N.; Zhu, L. Y.; Li, F. Y. Near-infrared photoregulated drug release in living tumor tissue via yolk-shell upconversion nanocages. Adv. Funct. Mater. 2014, 24, 363-371.

[145] Hegazy, M.; Zhou, P.; Wu, G. Y.; Wang, L.; Rahoui, N.; Taloub, N.; Huang, X.; Huang, Y. D. Construction of polymer coated core-shell magnetic mesoporous silica nanoparticles with triple responsive drug delivery. Polym. Chem. 2017, 8, 5852-5864.

[146] Peralta, M. E.; Jadhav, S. A.; Magnacca, G.; Scalarone, D.; Mártire, D. O.; Parolo, M. E.; Carlos, L. Synthesis and in vitro testing of thermoresponsive polymer-grafted core-shell magnetic mesoporous silica nanoparticles for efficient controlled and targeted drug delivery. J. Colloid Interface Sci. 2019, 544, 198-205.

[147] Yu, F.; Wu, H. J.; Tang, Y.; Xu, Y. F.; Qian, X. H.; Zhu, W. P. Temperature-sensitive copolymer-coated fluorescent mesoporous silica nanoparticles as a reactive oxygen species activated drug delivery system. Int. J. Pharm. 2018, 536, 11-20.

[148] Chang, B. S.; Sha, X. Y.; Guo, J.; Jiao, Y. F.; Wang, C. C.; Yang, W. L. Thermo and $\mathrm{pH}$ dual responsive, polymer shell coated, magnetic mesoporous silicananoparticles for controlled drug release. $J$. Mater. Chem. 2011, 21, 9239-9247.

[149] Zhao, J. J.; Zhang, P. H.; He, Z. M.; Min, Q. H.; Abdel-Halim, E. S.; Zhu, J. J. Thermal-activated nanocarriers for the manipulation of cellular uptake and photothermal therapy on command. Chem. Commun. 2016, 52, 5722-5725.

[150] Song, Y. H.; Li, D. D.; Lu, Y.; Jiang, K.; Yang, Y.; Xu, Y. J.; Dong, L.; Yan, X.; Ling, D. S.; Yang, X. Z. et al. Ferrimagnetic mPEG- $b$-PHEP copolymer micelles loaded with iron oxide nanocubes and emodin for enhanced magnetic hyperthermiachemotherapy. Natl. Sci. Rev. 2020, 7, 723-736.

[151] Ali, I.; Chen, L. M.; Huang, Y. J.; Song, L. P.; Lu, X. F.; Liu, B. Q.; Zhang, L.; Zhang, J. W.; Hou, L. X.; Chen, T. Humidity-responsive gold aerogel for real-time monitoring of human breath. Langmuir 2018, 34, 4908-4913.

[152] Zhu, Y. F.; Shi, J. L.; Li, Y. S.; Chen, H. R.; Shen, W. H.; Dong, X. P. Hollow mesoporous spheres with cubic pore network as a potential carrier for drug storage and its in vitro release kinetics. $J$. Mater. Res. 2005, 20, 54-61.

[153] Hou, Q.; Tao, X.; Yang, Y. J.; Ma, Y. Optimal synthesis of mesostructured hollow titania nanotubes templated on $\mathrm{CaCO}_{3}$ nanoparticles. Powder Technol. 2010, 198, 429-434.

[154] Qian, K.; Shi, T. Y.; He, S.; Luo, L. X.; Liu, X. L.; Cao, Y. S. Release kinetics of tebuconazole from porous hollow silica nanospheres prepared by miniemulsion method. Microporous Mesoporous Mater. 2013, 169, 1-6.

[155] Gong, L.; Sun, Y. Y.; Yu, M.; Gao, Y.; Zou, M. J.; Cheng, G. Development and evaluation of compression coating gastro-floating tablet of alfuzosin hydrochloride for zero-order controlled release. AAPS PharmSciTech 2018, 19, 3277-3286.

[156] Abhishek S, D.; Prashant J, G.; Abhijit A, A.; B. M, N. Sustained release dosage form: A concise review. Int. J. Pharm. Drug Anal. 2017, 5, 153-160. 
[157] Liu, J.; Qiao, S. Z.; Budi Hartono, S.; Lu, G. Q. Monodisperse yolk-shell nanoparticles with a hierarchical porous structure for delivery vehicles and nanoreactors. Angew. Chem., Int. Ed. 2010, 49, 4981-4985.

[158] Zhao, S. S.; Zhang, S. L.; Ma, J.; Fan, L.; Yin, C.; Lin, G.; Li, Q. Double loaded self-decomposable $\mathrm{SiO}_{2}$ nanoparticles for sustained drug release. Nanoscale 2015, 7, 16389-16398.

[159] Fan, L.; Jin, B. Q.; Zhang, S. L.; Song, C. J.; Li, Q. Stimuli-free programmable drug release for combination chemotherapy. Nanoscale 2016, 8, 12553-12559.

[160] Ding, Y. X.; Liu, J. J.; Li, X.; Xu, L. L.; Li, C.; Ma, L.; Liu, J. F.; Ma, R. J.; An, Y. L.; Huang, F. et al. Rational design of drug delivery systems for potential programmable drug release and improved therapeutic effect. Mater. Chem. Front. 2019, 3, 1159-1167.

[161] Zhang, S.; Chen, J.; Yu, Y. M.; Dai, K.; Wang, J.; Liu, C. S. Accelerated bone regenerative efficiency by regulating sequential release of BMP-2 and VEGF and synergism with sulfated chitosan. ACS Biomater. Sci. Eng. 2019, 5, 1944-1955.

[162] Nie, H. M.; Dong, Z. G.; Arifin, D. Y.; Hu, Y.; Wang, C. H. Core/shell microspheres via coaxial electrohydrodynamic atomization for sequential and parallel release of drugs. J. Biomed. Mater. Res. Part A 2010, 95A, 709-716.

[163] Ruiz-Esparza, G. U.; Wu, S. H.; Segura-Ibarra, V.; Cara, F. E.; Evans, K. W.; Milosevic, M.; Ziemys, A.; Kojic, M.; MericBernstam, F.; Ferrari, M. et al. Polymer nanoparticles encased in a cyclodextrin complex shell for potential site- and sequencespecific drug release. Adv. Funct. Mater. 2014, 24, 4753-4761.

[164] Dou, D. D.; Zhou, G.; Liu, H. W.; Zhang, J.; Liu, M. L.; Xiao, X. F.; Fei, J. J.; Guan, X. L.; Fan, Y. B. Sequential releasing of VEGF and BMP-2 in hydroxyapatite collagen scaffolds for bone tissue engineering: Design and characterization. Int. J. Biol. Macromol. 2019, 123, 622-628.

[165] Cong, Y.; Li, Q. J.; Chen, M.; Wu, L. M. Synthesis of dual-stimuliresponsive microcontainers with two payloads in different storage spaces for preprogrammable release. Angew. Chem., Int. Ed. 2017, $56,3552-3556$.

[166] Xu, W. N.; Ledin, P. A.; Iatridi, Z.; Tsitsilianis, C.; Tsukruk, V. V. Multicompartmental microcapsules with orthogonal programmable two-way sequencing of hydrophobic and hydrophilic cargo release. Angew. Chem., Int. Ed. 2016, 55, 4908-4913. 